\title{
Swarm Learning as a privacy-preserving machine learning approach for disease classification
}

\begin{abstract}
Stefanie Warnat-Herresthal ${ }^{1, *}$, Hartmut Schultze $e^{2, *}$, Krishnaprasad Lingadahalli Shastry ${ }^{2, *}$, Sathyanarayanan Manamohan², Saikat Mukherjee ${ }^{2}$, Vishesh Garg², Ravi Sarveswara², Kristian Händler ${ }^{3,{ }^{*}}$, Peter Pickkers ${ }^{4, *}$, N. Ahmad Aziz ${ }^{5,6, *}$, Sofia Ktena ${ }^{7,{ }^{*}}$ Christian Siever ${ }^{2}$, Michael Kraut ${ }^{3}$, Milind Desai ${ }^{2}$, Bruno Monnet ${ }^{2}$, Maria Saridaki ${ }^{7}$, Charles Martin Siegel ${ }^{2}$, Anna Drews $^{3}$, Melanie Nuesch-Germano ${ }^{1}$, Heidi Theis ${ }^{3}$, Mihai G. Netea ${ }^{8,9}$, Fabian Theis ${ }^{10}$, Anna C. Aschenbrenner ${ }^{1,8}$, Thomas Ulas ${ }^{3}$, Monique M.B. Breteler,11,\#, Evangelos J. GiamarellosBourboulis $^{7, \#}$, Matthijs Kox ${ }^{4, \#}$, Matthias Becker ${ }^{3, \#}$, Sorin Cheran", ${ }^{2, \#}$, Michael S. Woodacre, ${ }^{2, \#}$, Eng Lim Goh",\#, Joachim L. Schultze ${ }^{1,3, \#, ~ G e r m a n ~ C O V I D-19 ~ O M I C S ~ I n i t i a t i v e ~(D e C O I) ~}$
\end{abstract}

\section{Affiliations:}

1 Genomics and Immunoregulation, Life \& Medical Sciences (LIMES) Institute, University of Bonn, 53115 Bonn, Germany

2 Hewlett Packard Enterprise

3 German Center for Neurodegenerative Diseases (DZNE), PRECISE Platform for Single Cell Genomics and Epigenomics at DZNE and the University of Bonn, 53175 Bonn, Germany

4 Department of Intensive Care Medicine and Radboud Center for Infectious Diseases (RCl), Radboud University Medical Center, Nijmegen, 6500HB, The Netherlands

5 Population Health Sciences, German Center for Neurodegenerative Diseases (DZNE), 53175 Bonn, Germany

6 Department of Neurology, Faculty of Medicine, University of Bonn, 53127 Bonn, Germany

7 4th Department of Internal Medicine, National and Kapodistrian University of Athens, Medical School, 12462 Athens, Greece

8 Department of Internal Medicine and Radboud Center for Infectious Diseases (RCI), Radboud University Medical Center, Nijmegen 6500HB, The Netherlands

9 Immunology \& Metabolism, Life and Medical Sciences (LIMES) Institute, University of Bonn, Bonn 53115, Germany

10 Institute of Computational Biology, Helmholtz Center Munich (HMGU), 85764 Neuherberg, Germany

11 Institute for Medical Biometry, Informatics and Epidemiology (IMBIE), Faculty of Medicine, University of Bonn, 53175 Bonn, Germany

* shared first authors

\# shared last authors

corresponding author: joachim.schultze@dzne.de

Key Words: 
bioRxiv preprint doi: https://doi.org/10.1101/2020.06.25.171009; this version posted June 29, 2020. The copyright holder for this preprint (which was not certified by peer review) is the author/funder, who has granted bioRxiv a license to display the preprint in perpetuity. It is made available under aCC-BY-NC-ND 4.0 International license.

48 machine learning, artificial intelligence, Swarm Learning, decentralized machine learning, 49 privacy preserving machine learning, transcriptomics, blood, blood transcriptomics, acute 50 myeloid leukemia, acute lymphoblastic leukemia, COVID-19, tuberculosis 


\section{$51 \quad$ Abstract}

52

53

54

55

56

57

Identification of patients with life-threatening diseases including leukemias or infections such as tuberculosis and COVID-19 is an important goal of precision medicine. We recently illustrated that leukemia patients are identified by machine learning (ML) based on their blood transcriptomes. However, there is an increasing divide between what is technically possible and what is allowed because of privacy legislation. To facilitate integration of any omics data from any data owner world-wide without violating privacy laws, we here introduce Swarm Learning (SL), a decentralized machine learning approach uniting edge computing, blockchain-based peer-to-peer networking and coordination as well as privacy protection without the need for a central coordinator thereby going beyond federated learning. Using more than 14,000 blood transcriptomes derived from over 100 individual studies with nonuniform distribution of cases and controls and significant study biases, we illustrate the feasibility of SL to develop disease classifiers based on distributed data for COVID-19, tuberculosis or leukemias that outperform those developed at individual sites. Still, SL completely protects local privacy regulations by design. We propose this approach to noticeably accelerate the introduction of precision medicine.

\section{Introduction}

Fast and reliable detection of patients with severe illnesses is a major goal of precision medicine ${ }^{1}$. The measurement of molecular phenotypes for example by omics technologies ${ }^{2}$ and the application of sophisticated bioinformatics including artificial intelligence (Al) approaches $^{3-7}$ opens up the possibility for physicians to utilize large-scale data for diagnostic purposes in an unprecedented way. Yet, there is an increasing divide between what is technically possible and what is allowed because of privacy legislation ${ }^{8}$ (hhs.gov, https://www.hhs.gov/hipaa/index.html, 2020; Intersoft Consulting, General Data Protection Regulation, https://gdpr-info.eu; Convention for the Protection of Individuals with regard to Automatic Processing of Personal Data, https://rm.coe.int/16808ade9d). Particularly, in a global health crisis, as in the case of the infection with severe acute respiratory syndrome coronavirus 2 (SARS-CoV-2) leading to the pandemic spread of coronavirus disease 2019 (COVID-19) ${ }^{9-11}$, reliable, fast, secure and privacy-preserving technical solutions based on Al principles are now believed to add to the armamentarium to quickly answer important questions in the fight against such threats ${ }^{12-15}$. These Al-based concepts range from protein 
structure prediction ${ }^{16}$, drug target prediction ${ }^{17}$, knowledge sharing ${ }^{18}$, tools for population control $^{19,20}$ to the assistance of healthcare personnel, e.g. by developing Al-based coronavirus diagnostic software ${ }^{21,22}$. Considering the more clinically oriented Al-based technical solutions, any such progress might also induce improvements for a variety of deadly diseases including other major infections or cancer ${ }^{23}$. For example, the principles of a recently introduced Alsystem for diagnosing COVID-19 pneumonia and predicting disease outcome using computed tomography 22 might be further developed to identify patients with tuberculosis or lung cancer in the future ${ }^{24}$. At the same time, we need to consider important standards relating to data privacy and protection, such as Convention 108(+) of the Council of Europe (Convention for the Protection of Individuals with regard to Automatic Processing of Personal Data, https://rm.coe.int/16808ade9d), which regulate the use and sharing of health data including in Al-based approaches, irrespective of the occurrence of a pandemic crisis.

Al-based solutions intrinsically rely on appropriate algorithms ${ }^{25}$, but even more so on large enough datasets for training purposes ${ }^{26}$. Since the domain of medicine is inherently decentralized, the volume of data available locally is often insufficient to train reliable classifiers $^{27-29}$. As a consequence, centralization of data, for example via cloud solutions, has been one model to address the local limitations ${ }^{30-32}$. While beneficial from an Al-perspective, centralized solutions were shown to have other inherent hurdles, including increased data traffic of large medical data, data ownership, privacy and security concerns when ownership is disconnected from access and usage curation and thereby creating data monopolies favoring data aggregators ${ }^{26}$. Consequently, solutions to the challenges of central data models in Al - particular when dealing with medical data - must be effective, with high accuracy and efficiency, privacy- and ethics-preserving, secure, and fault-tolerant by design ${ }^{33-36}$. Federated $\mathrm{Al}$ has been introduced to address some of these aspects ${ }^{26,37-39}$. While data are kept locally (at the edge) and privacy issues are addressed ${ }^{40,41}$, the model parameters in federated $\mathrm{Al}$ are still handled by central custodians who as the intermediaries concentrate power of the learning to themselves. Furthermore, such star-shaped architectures decrease fault tolerance.

We hypothesized that completely decentralized Al solutions overcome current technical shortcomings and at the same time accommodate for inherently decentralized data structures in medicine as well as pronounced data privacy and security regulations. The solution would 1) need to keep large medical data locally with the data owner, 2) require no raw data exchange thereby also reducing data traffic and issues related to central storage, 3) provide high level data security and privacy protection, 4) guarantee secure, transparent and fair onboarding of decentralized members participating in the learning network without the need for a central custodian, 5) allow for parameter merging with equal rights for all members requiring no central custodian, and 6 ) protect the $M L$ models from attacks. To address these 
120 points, we introduce the concept of Swarm Learning (SL). SL combines decentralized

121 hardware infrastructures, distributed ML technique based on standardized Al engines with a

122 permissioned blockchain to securely onboard members, dynamically elect the leader among

123 the members, and merge model parameters. All processes are orchestrated by an SL library

124 and an iterative learning procedure applying Al solutions to compute problems with 125 decentralized private data.

126 Medicine is a prime example to illustrate the advantages of this Al approach. Without any 127 doubt, numerous medical features including radiograms or computed tomographies, 128 proteomes, metagenomes or microbiomes derived from body fluids including nasal or throat 129 swaps, blood, urine or stool are all excellently suitable medical data for the development of Al130 based diagnostic or outcome prediction classifiers. We here chose to evaluate the cellular 131 compartment of peripheral blood, either in form of peripheral blood mononuclear cells (PBMC) 132 or whole blood-derived transcriptomes, since blood-derived transcriptomes include important 133 information about the patients' immune response during a certain disease, which in itself is an 134 important molecular information ${ }^{42,43}$. In other words, in addition to the use of blood-derived 135 high-dimensional molecular features for a diagnostic or outcome classification problem, blood 136 transcriptomes could be further utilized in the clinic to systematically characterize ongoing 137 pathophysiology, predict patient-specific drug targets and trigger additional studies targeting 138 defined cell types or molecular pathways, making this feature space even more attractive to 139 answer a wide variety of medical questions. Here, we illustrate that newly generated blood 140 transcriptome data together with data derived from more than 14,000 samples in more than 141100 studies combined with Al-based algorithms in a Swarm Learning environment can be 142 successfully applied in real-world scenarios to detect patients with leukemias, tuberculosis or 143 active COVID-19 disease in an outbreak scenario across distributed datasets without the necessity to negotiate and contractualize data sharing.

\section{Results}

\section{Concept of Swarm Learning}

149 Machine learning (ML) of any data including genome or transcriptome data requires the 150 availability of sufficiently large datasets ${ }^{26,44}$ and the respective compute infrastructure including 151 data storage for data processing and analytics ${ }^{45}$. Conceptually, if data and compute 152 infrastructure is sufficiently available locally, ML can be performed locally ('at the edge') (Fig. 153 1a). However, often medical data are not sufficiently large enough locally and similar 
approaches are performed at different locations in a disconnected fashion. These limitations have been overcome by cloud computing where data are moved centrally to perform training of $\mathrm{ML}$ algorithms in a centralized compute environment (Fig. 1b). Compared to local approaches, cloud computing can significantly increase the amount of data for training $\mathrm{ML}$ algorithms and therefore significantly improve their results ${ }^{26}$. However, cloud computing has other disadvantages such as data duplication from local to central data storage, increased data traffic and issues with locally differing data privacy and security regulations ${ }^{46}$. As an alternative, federated cloud computing approaches such as Google's federated learning ${ }^{38}$ and Facebook's elastic averaging SGD (Deep learning with Elastic Averaging SGD, http://papers.neurips.cc/paper/5761-deep-learning-with-elastic-averaging-sgd.pdf) have been developed. In these models, dedicated parameter servers are responsible for aggregating and distributing local learning (Fig. 1c). A disadvantage of such star-shaped system architectures is the remainder of a central structure, which hampers implementation across different jurisdictions and therefore still requires the respective legal negotiations. Furthermore, the risk for a single point of failure at the central structure reduces fault-tolerance.

In an alternative model, which we introduce here as Swarm Learning (SL), we dismiss the dedicated server and allow parameters and models to be shared only locally (Fig. 1d). While parameters are shared via the swarm network, the models are built independently on private data at the individual sites, here referred to as swarm edge nodes (short 'nodes') (Fig. 1e). SL provides security measures to guarantee data sovereignty, security and privacy realized by a private permissioned blockchain technology which enables different organizations or consortia to efficiently collaborate (Fig. 1f). In a private permissioned blockchain network, each participant is well defined and only pre-authorized participants can execute the transactions. Hence, they use computationally inexpensive consensus algorithms, which offers better performance and scalability. Onboarding of new members or nodes can be done dynamically with the appropriate authorization measures to know the participants of the network, which allows continuous scaling of learning (Extended Data Fig. 1a). A new node enrolls via a blockchain smart contract, obtains the model, and performs local model training until defined conditions for synchronization are met. Next, model parameters are exchanged via a Swarm API with the rest of the swarm members and merged for an updated model with updated parameter settings to start a new round of training at the nodes. This process is repeated until stopping criterions are reached, which are negotiated between the swarm nodes/members. The leader is dynamically elected using a blockchain smart contract for merging the parameters and there is no need for a central coordinator in this swarm network. The parameter merging algorithm is executed using a blockchain smart contract thus protects it from semi-honest or dishonest participants. The parameters can be merged by the leader 
using different functions including average, weighted average, minimum, maximum, or median functions. The various merge techniques and merge frequency enables SL to efficiently work with imbalanced and biased data. As currently developed, SL works with parametric models with finite sets of parameters, such as linear regression or neural network models.

At each node, SL is conceptually divided into infrastructure and application layer (Fig. 19). On top of the physical infrastructure layer (hardware) the application environment contains the $\mathrm{ML}$ platform, the blockchain, and the SL library (SLL) including the Swarm API in a containerized deployment, which allows SL to be executed in heterogeneous hardware infrastructures (Fig. 1g, Supplementary Information). The application layer consists of the content, the models from the respective domain, here medicine (Fig. 1g), for example blood transcriptome data from patients with leukemias, tuberculosis and COVID-19 (Fig. 1h-I). Collectively, Swarm Learning allows for a completely decentralized and therefore democratized, secure, privacypreserving, hardware-independent, and scalable machine learning environment, applicable to many scenarios and domains, which we demonstrate with three medical examples.

\section{Swarm learning robustly predicts leukemias from peripheral blood mononuclear cell} data

As a first use case, we chose transcriptomes derived from peripheral blood mononuclear cells (PBMC) of more than 12,000 individuals (Fig. 1 h-j) separated into three individual datasets (A1, A2, A3) based on the technology used for generating the transcriptomes (2 different microarrays, RNA-seq) ${ }^{47}$. We used a deep neural network (Keras, https://keras.io/, 2015) as the machine learning approach in all three use cases. To assess performance metrics of SL, we simulated scenarios by dividing up the individual samples derived from several independently performed studies (see Material and Methods) within each of the three datasets into non-overlapping training and test sets. The training sets were then distributed to three nodes for training and classifiers were tested at a fourth node (independent test set) (Fig. 2a). By assigning the training data to the nodes in different distributions, we mimicked several clinically relevant scenarios (Supplementary Table 1). As cases, we first used samples defined as acute myeloid leukemia (AML), all other samples are termed 'controls'. Each node within this simulation could stand for a large hospital or center, a network of hospitals performing individual studies together, a country or any other independent institutional organization generating such medical data with local privacy requirements.

In a first scenario, we randomly distributed samples per node as well as cases and controls unevenly at the nodes and between nodes (dataset A2) (Fig. 2b). Sample distribution between sample sets was permuted 100 times (Fig. 2b, middle panel) to determine the influence of 
individual samples on overall performance. Among the nodes, the best test results were obtained by node one with a mean accuracy of $97.0 \%$, mean sensitivity of $97.5 \%$ and mean specificity of $96.3 \%$ with an even distribution between cases and controls, albeit this node had the smallest number of overall training samples. Node 2 did not produce any meaningful results, which was due to a too low ratio of cases to controls (1:99) for training. Surprisingly, node 3 with the largest number of samples, but an uneven distribution (70\% cases : $30 \%$ controls) performed worse than node 1 with a mean balanced accuracy of $95.1 \%$. Most importantly, however, SL outperformed each of the nodes resulting in a higher test accuracy in $97.0 \%$ of all permutations (mean balanced accuracy $97.7 \%$ ) (Fig. 2b, right panel,

\section{Supplementary Table 4). The balanced accuracy of SL was significantly higher $(p<0.001)$} when compared to the performance of each of the three nodes, despite the fact that information from the poorly performing node 2 was integrated. We also calculated this scenario in datasets $\mathrm{A} 1$ and $\mathrm{A} 3$ and obtained rather similar results strongly supporting that the performance improvement of SL over single nodes is independent of data collection (studies) and even experimental technologies (microarray (datasets A1, A2), RNA-seq (dataset A3) used for data generation (Extended Data Fig. 2).

To test whether more evenly distributed samples at the nodes would improve individual node performance, we distributed similar numbers of samples to each of the nodes but kept case:control ratios as in scenario 1 (Fig. 2c, Extended Data Fig. 3). While there was a slight increase in test accuracy at nodes 1 and 2 , node 3 performed worse with also higher variance. More importantly, SL still resulted in the best performance metrics (mean $98.5 \%$ accuracy) with slightly but significantly ( $p<0.001$ ) increasing performance compared to the first scenario. Results derived from datasets A1 and A3 echoed these findings (Extended Data Fig. 3).

In a third scenario, we distributed the same number of samples across all three nodes, but increased potential batch effects between nodes, by distributing samples of a clinical study independently performed and published in the past only to a dedicated training node. In this scenario, cases and control ratios varied between nodes and left out samples (independent samples) from the same published studies were combined for testing at node 4 . Performance of the three nodes was very comparable, but never reached SL results (mean $98.3 \%$ accuracy, swarm outperformed all nodes with $p<0.001$, Fig. 2d., Extended Data Fig. 4b, Supplementary Data Table 4), which was also true for datasets A1 and A3 (Extended Data Fig. 4c-d). Even when further increasing batch effects by distributing samples from independent published studies to the test node, which means that training and test datasets come from studies performed and published independently, SL outperformed the individual nodes, albeit the variance in the results was increased both at each node and for SL, indicating 
that study design has an overall impact on classifier performance and that this is still seen in SL (mean 95.6\% accuracy, Extended Data Fig. 4e).

In a fourth scenario, we further optimized the nodes by increasing the overall sample size at node 3 and keeping case:control ratios even at all nodes (Fig. 2e, Extended Data Fig. 5a-d). Clearly, node performance further improved with little variance between permutations, however, even under these 'node-optimized' conditions, SL led to higher performance parameters.

In a fifth scenario, we tested whether or not SL was 'immune' against the impact of the data generation procedure (microarray versus RNA-seq) (Fig. 2f, Extended Data Fig. 5e,f). We recently demonstrated that classifiers trained on data derived by one technology (e.g. microarrays) do not necessarily perform well on another (e.g. RNA-seq) ${ }^{47}$. To test this influence on SL, we distributed the samples from the three different datasets (A1-A3) to one node each, e.g. dataset $A 1$ was used for training only at node 1 . We used $20 \%$ of the data (independent non-overlapping to the training data) from each dataset (A1-A3) and combined them to form the test set (node 4). Node 3, trained on RNA-seq data, performed poorly on the combined dataset due to the fact that two-thirds of the data in the test set were microarrayderived data. Nodes 1 and 2 performed reasonably well with mean accuracies of $96.1 \%$ (node 1) and $97.5 \%$ (node 2), however did not reach the test accuracy of SL (98.8\%), which also indicated that SL is much more robust toward effects introduced by different data production technologies in transcriptomics (Fig. 2f, Extended Data Fig. 5e,f).

Finally, we repeated several of these scenarios with acute lymphoblastic leukemia (ALL) as the second most prevalent disease in dataset A2 (Extended Data Fig. 6 and data not shown) and demonstrated very similar results with SL outperforming the classifiers built at the nodes. Collectively, these simulations using real-world transcriptome data collected from more than 100 individual studies illustrate that SL would not only allow data to be kept at the place of generation and ownership, but it also outperforms every individual node in numerous scenarios, even in those with nodes included that cannot provide any meaningful classifier results.

\section{Swarm learning to identify patients with tuberculosis}

290 In infectious diseases, heterogeneity may be more pronounced compared to leukemia, 291 therefore we built a second use case predicting cases with tuberculosis (Tb) from full blood 292 transcriptomes. Of interest, previous work in smaller studies had already suggested that acute 293 tuberculosis or outcome of tuberculosis treatment can be revealed by blood transcriptomics 
48-52. To apply SL, we generated a new dataset based on full blood transcriptomes derived by PaxGene blood collection followed by bulk RNA-sequencing. We also generated new blood transcriptomes and added existing studies to the dataset compiling a total of 1,999 samples from nine individual studies including 775 acute and 277 latent Tb cases (Fig. 1k, Extended Data Fig. 7a, Supplementary Table 2). These data are more challenging, since infectious diseases show more variety due to biological differences with respect to disease severity, phase of the disease or the host response. But also the technology itself is more variable with numerous different approaches for full blood transcriptome sample processing, library production and sequencing, which can introduce technical noise and batches between studies. As a first scenario, we used all Tb samples (latent and acute) as cases and divided Tb cases and controls evenly among the nodes (Extended Data Fig. S7a-b, Supplementary Table 1). Similar to $A M L$ and $A L L$, in detecting $T b, S L$ outperformed the individual nodes in accuracy (mean 93.4\%), sensitivity (mean 96.0\%) and specificity (mean 90.9\%) (Extended Data Fig. S7b). To increase the challenge, we decided to assess prediction of acute Tb cases only. In this scenario, latent Tb are not treated as cases but rather as controls (Extended Data Fig. S7a). For the first scenario, we kept cases and controls even at all nodes but further reduced the number of training samples (Fig. 3a-b). As expected in this more challenging scenario, distinguishing acute Tb from the control cohort (including latent Tb samples), overall performance (mean balanced accuracy $89.1 \%$, mean sensitivity $92.2 \%$, mean specificity $86.0 \%)$ slightly dropped, but still SL performed better than any of the individual nodes $(p<0.01$ for swarm vs. each node, Fig. 3b). To determine whether sample size impacts on prediction results in this scenario, we reduced the number of samples at each training node (1-3) by $50 \%$, but kept the ratio between cases and controls (Extended Data Fig. S7c). Still, SL outperformed the nodes, but all statistical readouts (mean accuracy $86.5 \%$, mean sensitivity $87.8 \%$, mean specificity $84.8 \%$ ) at all nodes and SL showed lower performance, following general observations of $\mathrm{Al}$ with better performance when increasing training data ${ }^{26}$. We next altered the scenario by dividing up the three nodes into six smaller nodes (Fig. 3c, samples per node reduced by half in comparison to Fig. 3a-b), a scenario that can be envisioned in the domain of medicine in many settings, for example if several smaller medical centers with less cases would join efforts (Fig. 3d). Clearly, each individual node performed worse, but for SL the results did not deteriorate (mean accuracy $89.2 \%$, mean sensitivity $90.7 \%$, mean specificity $88.2 \%$ with significant difference to each of the nodes in all performance measures, see Supplementary Table 4), again illustrating the strength of the joined learning effort, while completely respecting each individual node's data privacy.

Albeit aware of the fact that - in general - acute Tb is an endemic disease and does not tend to develop towards a pandemic such as the current COVID-19 pandemics, we utilized the $\mathrm{Tb}$ 
330 blood transcriptomics dataset to simulate potential outbreak and epidemic scenarios to 331 determine benefits, but also potential limitations of SL and how to address them (Fig. 3e-I).

332 The first scenario reflects a situation in which three independent regions (simulated by the 333 nodes), would already have sufficient but different numbers of disease cases. Furthermore, 334 cases and controls were kept even at the test node (Fig. 3e-f). Overall, compared to the 335 scenario described in Fig. 3c, results for the swarm were almost comparable (mean accuracy $33689.0 \%$, mean sensitivity $94.4 \%$, mean specificity $83.4 \%$ ), while the results for the node with the lowest number of cases and controls (node 2) dropped noticeable (mean accuracy $82.2 \%$, mean sensitivity $88.8 \%$, mean specificity $75.4 \%$, Fig. 3f). When reducing the prevalence at the test node by increasing the number of controls (Fig. $\mathbf{3 g}-\mathbf{h}$ ), this effect was even more pronounced, while the performance of the swarm was almost unaffected (mean balanced accuracy $89.0 \%)$.

We decreased the number of cases at a second training node (node 1) (Fig. 3i-I), which clearly reduced test performance for this particular node (Fig. 3i-j), while test performance of the swarm was only slightly inferior to the prior scenario (mean balanced accuracy $87.5 \%$, no significant difference to the prior scenario). Only when reducing the prevalence at the test node (Fig. 3k-I), we saw a further drop in mean specificity for the swarm (81.0\%), while sensitivity stayed similarly high (93.0\%). Finally, we further reduced the prevalence at two training nodes (node 2: 1:10; node 3: 1:5) as well as the test node (Extended Data Fig. 8ab). Lowering the prevalence during training resulted in very poor test performance at these two nodes (balanced accuracy node 2: $59.8 \%$, balanced accuracy node 3: $74.8 \%$ ), while specificity was high (node 2: $98.4 \%$, node 3: 93.8\%). SL showed highest accuracy (mean balanced accuracy $86.26 \%$ ) and F-statistics (90.0\%) but was outperformed for sensitivity by node 1 (swarm: $80.0 \%$, node1: 87.8\%), which showed poor performance concerning specificity (swarm: $92.4 \%$, node1: 84.8\%). Vice versa, node 2 outperformed the swarm for specificity (98.4\%), but showed very poor sensitivity (21.2\%) (Extended Data Fig. 8b). When lowering prevalence at the test node (Extended Data Fig. 8c-d), it became clear that all performance parameters including the $\mathrm{F} 1$ statistics were more resistant for the swarm compared to individual nodes. Taken together, using whole blood transcriptomes instead of PBMC and acute Tb as the disease instead of leukemia, we present a second use case illustrating that Swarm Learning integrating several individual nodes outperforms each node. Furthermore, we gained initial insights into the potential of SL to be utilized in a disease outbreak scenario. 
Based on the promising results obtained for tuberculosis, we collected blood from COVID-19 patients at two sites in Europe (Athens, Greece; $n=39$ samples, Nijmegen, $n=93$ samples) and generated whole blood transcriptomes by RNA-sequencing. We used the dataset described for $\mathrm{Tb}$ as the framework and included the COVID-19 samples (Fig. 1I) for assessing whether SL could be applied early on to detect patients with a newly identified disease. While COVID19 patients are currently identified by PCR-based assays to detect viral RNA ${ }^{53}$, we use this case as a proof-of-principle study to illustrate how SL could be used even very early on during an outbreak based on the patients' immune response captured by analysis of the circulating immune cells in the blood. Here, blood transcriptomes only present a potential feature space to illustrate the performance of SL. Furthermore, assessing the specific host response, in addition to disease prediction, might be beneficial in situations for which the pathogen is unknown, specific pathogen tests not yet possible, and blood transcriptomics can contribute to the understanding of the host's immune response ${ }^{54}$. Lastly, while we do not have the power yet, blood transcriptome-based machine learning might be used to predict severe COVID-19 cases, which cannot be done by viral testing alone.

COVID-19 induces very strong changes in peripheral blood transcriptomes ${ }^{54}$. Following our experience with the leukemia and tuberculosis use cases, we first tested classifier performance for evenly distributed cases and controls at both training nodes and the test node (Extended Data Fig. 9a,b, Supplementary Table 1). We reached very high statistical performance parameters, including high F1-statistics with SL showing highest mean values for accuracy (96.4\%), sensitivity (97.8\%), and F1 score (96.4\%) (Extended Data Fig. 9b, summary statistics for all figures are listed in Supplementary Table 4). Reducing the prevalence at the test node (11:25 cases:controls) reduced all test parameters (Extended Data Fig. 9c), but only when we reduced the prevalence even further (1:44 ratio, Extended Data Fig. 9d), F1-statistics was clearly reduced, albeit SL again performing best. We next reduced the cases at all training nodes (Extended Figure 10), but even under these conditions, we observed still very high values for accuracy, sensitivity, specificity and F1 scores, both derived by training at individual nodes or by SL (Extended Figure 10a-f).

We then reduced the cases at all three training nodes to very low numbers, a scenario that might be envisioned very early during an outbreak scenario (Fig. 4a). Node 1 contained only 20 cases, node 210 cases and node 3 only 5 cases. At each node, controls outnumbered cases by 1:5, 1:10, or 1:20. At the test node, we varied the prevalence from 1:1 (Fig. 4b), 1:2 (Fig. 4c) to 1:10 (Fig. 4d). Based on our findings for Tb (Extended Data Fig. 8), we expected classifier performance to deteriorate under these conditions. We only observed decreased performance at nodes 2 and 3 in these scenarios with SL outperforming these nodes with 
401 (99.3\%), sensitivity (95.1\%), specificity (99.7\%) and F1-statistics (99.7\%) (Fig. 4d). Finally,

402 we simulated a scenario with four instead of three training nodes with very few cases per node

403 (Extended Data Fig. 11a-d), in an otherwise similar scenario as described for Fig. 4. Even for

404 a simulated prevalence of 1:10 cases versus controls at the test node, we determined high

405 test performance parameters for SL, with swam performance being significantly higher than

406 node performances (SL accuracy (99.1\%), sensitivity (92.0\%), specificity (99.9\%), F1 statistics

407 (99.7\%) (Extended Data Fig. 11) with the lowest variance in performance, while results at

408 individual notes were very variable and deteriorated with low case numbers at the training

409 node. Collectively, we provide first evidence that blood transcriptomes taken from patients with

410 COVID-19 harbor very strong biological changes and these translate into a very powerful

411 feature space for applying machine learning to the detection of patients with this new infectious

412 disease, particularly when applying SL.

\section{Discussion}

415 The introduction of precision medicine based on high-resolution molecular and imaging data

416 will heavily rely on trustworthy machine learning algorithms in compute environments that are

417 characterized by high accuracy and efficiency, that are privacy- and ethics-preserving, secure,

418 and that are fault-tolerant by design ${ }^{33-36}$. At the same time, privacy legislation is becoming 419 increasingly strict, as risks of cloud-based and central data-acquisition are recognized. Here, 420 we introduce Swarm Learning, which combines blockchain technology and machine learning 421 environments organized in a swarm network architecture with independent swarm edge nodes 422 that harbor local data, compute infrastructure and execute the shared learning models that 423 make central data acquisition obsolete. During iterations of SL, one of the nodes is chosen to 424 lead the iteration, which does not require a central parameter server anymore thereby 425 restricting centralization of learned knowledge and at the same time increasing resiliency and 426 fault tolerance. In fact, these are the most important improvements over current federated 427 computing models. Furthermore, private permissioned blockchain technology, harboring all 428 rules of interaction between the nodes, is the Swarm Learning's inherent privacy- and ethics429 preserving strategy. This is of particular interest to medical data and could be adapted by other 430 federated learning systems. To understand whether the concept of swarm learning would also 431 be characterized by high efficiency and high accuracy, we built three medical use cases based 432 on blood transcriptome data, which are high-dimensional data derived from blood, one of the 433 major tissues used for diagnostic purposes in medicine. First, utilizing three previously 434 compiled datasets (A1-3) of peripheral blood mononuclear cells derived from patients with 435 acute myeloid leukemia, we provide strong evidence that SL-based classifier generation using 
a well-established neural network algorithm outperforms individual nodes, even in scenarios where individual contributing swarm nodes were performing rather poorly. Most striking, swarm learning was even improving performance parameters when training of individual nodes was based on technically different data, a situation that was previously shown to deteriorate classifier performance ${ }^{47}$. With these promising results, we generated a more challenging use case in infectious disease patients, detecting $\mathrm{Tb}$ based on full blood transcriptomes. Also in this case, SL outperformed individual nodes. Using Tb to simulate scenarios that could be envisioned for building blood transcriptome classifiers for patients during an outbreak situation, we further illustrate the power of SL over individual nodes. Considering the difficulty to quickly negotiate data sharing protocols or contracts during an epidemic or pandemic outbreak, we deduce from these findings that SL would be an ideal strategy for independent producer of medical data to quickly team up to increase the power to generate robust and reliable machine learning-based disease or outcome prediction classifier without the need to share data or relocate data to central cloud storages.

In addition, we tested whether we could build a disease prediction classifier for COVID-19 in an outbreak scenario. Building on our knowledge that blood transcriptomes of COVID-19 patients are significantly altered with hundreds of genes being changed in expression and with a rather specific signature compared to other infectious diseases ${ }^{54}$, we hypothesized that it should be possible to build such a classifier with a rather small number of samples. Here, we provide evidence that classifiers with high accuracy, sensitivity, specificity, and also high F1statistics can be generated to identify patients with COVID-19 based on their blood transcriptomes. Moreover, we illustrate the power of SL that would allow to quickly increase the power of classifier generation even under very early outbreak scenarios with very few cases used at the training nodes, which could be e.g. collaborating hospitals in an outbreak region. Since data do not have to be shared, additional hospitals could benefit from such a system by applying the classifiers to their new patients and once classified, one could even envision an onboarding of these hospitals for an adaptive classifier improvement schema. Albeit technically feasible, we are fully aware that such scenarios require further classifier testing and confirmation, but also an assessment of how this could be integrated in existing legal and ethical regulations at different regions in the world ${ }^{5,6}$. Furthermore, we appreciate that other currently less expensive data might be suitable for generating classifiers to identify COVID-19 patients ${ }^{10}$. For example, if highly standardized clinical data would become available, SL could be used to interrogate the clinical feature space at many clinics worldwide without any need to exchange the data to develop high performance classifiers for detecting COVID-19 patients. Similarly, recently introduced Al-systems using imaging data ${ }^{21,22}$ might be 
of these additional opportunities using other parameter spaces, we would like to suggest blood transcriptomics as a promising new alternative due to its very strong signal in COVID-19. A next step will be to determine whether blood transcriptomes taken at early time points could be used to predict severe disease courses, which might allow physicians to introduce novel treatments at an earlier time point. Furthermore, we propose to develop an international database of blood transcriptomes that could be utilized for the development of predictive classifiers in other infectious and non-infectious diseases as well. It could be envisioned that such an SL-based learning scheme could be deployed as a permanent monitoring or early warning system that runs by default, looking for unusual movements in molecular profiles. Collectively, SL together with transcriptomics but also other medical data is a very promising approach to democratize the use of Al among the many stakeholders in the domain of medicine while at the same time resulting in more data privacy, data protection and less data traffic.

With increasing efforts to enforce data privacy and security of medical data ${ }^{8}$ (hhs.gov, https://www.hhs.gov/hipaa/index.html, 2020; Intersoft Consulting, General Data Protection Regulation, https://gdpr-info.eu) and to reduce data traffic and duplication of large medical data, a decentralized data model will become the preferred choice of handling, storing, managing and analyzing medical data ${ }^{26}$. This will not be restricted to omics data as exemplified here, but will extend to other large medical data such as medical imaging data ${ }^{55,56}$. Particularly in oncology, great successes applying machine learning have already been reported for tumor detection $47,55,57,58$, subtyping 59,60 , grading ${ }^{61}$, genomic characterization ${ }^{62}$, or outcome prediction $^{63}$, yet progress is hindered by too small datasets at any given institution ${ }^{26}$ with current privacy regulations ${ }^{8}$ (hhs.gov, https://www.hhs.gov/hipaa/index.html, 2020; Intersoft Consulting, General Data Protection Regulation, https://gdpr-info.ee) making it less appealing to develop centralized Al systems. We introduce Swarm Learning as a decentralized learning system with access to data stored locally that can replace the current paradigm of data sharing and centralized storage while preserving data privacy in cross-institutional research in a wide spectrum of biomedical disciplines. Furthermore, SL can easily inherit developments to further preserve privacy such as functional encryption ${ }^{64}$, or encrypted transfer learning approaches ${ }^{65}$.

501 In addition, the blockchain technology applied here provides robust measures against semihonest or dishonest participants/adversaries who might attempt to undermine a Swarm

503 Network. Another important aspect for wide employment of SL in the research community and 504 in real-world applications is the ease of use of the Swarm API, which will make it easier for 505 researchers and developers to include novel developments such as for example private machine learning in TensorFlow ${ }^{66}$. 
507 There is no doubt that numerous medical and other data types as well as a vast variety of 508 computational approaches can be used during a pandemic ${ }^{14}$. We do not want to imply that 509 blood transcriptomics would be the preferred solution for the many questions that $\mathrm{Al}$ and 510 machine learning could help to solve during such a crisis. Although, at the same time, we have 511 recently shown that blood transcriptomics can be used to define molecular phenotypes of 512 COVID-19, uncover the deviated immune response in severe COVID-19 patients, define 513 unique patterns of the disease in comparison to other diseases and can be utilized to predict 514 potential drugs to be repurposed for COVID-19 therapy (Aschenbrenner et al. unpublished 515 results). Therefore, we explored blood transcriptomics as a unique and rich feature space and 516 a good example to illustrate the advantages of SL in identifying COVID-19 patients. Once 517 larger datasets become available, SL could be used to identify patients at risk to develop 518 severe COVID-19 early after onset of symptoms.

519 Another important quest that has been proposed is global collaboration and data-sharing ${ }^{13}$. 520 While we could not agree more about the need for global collaboration - an inherent 521 characteristic of SL - we favor systems that do not require data sharing but rather support 522 global collaboration with complete data privacy preservation. Particularly, if using medical data 523 that can also be used to interrogate medical issues unrelated to COVID-19. Indeed, 524 statements by lawmakers have been triggered, clearly indicating that privacy rules also fully 525 apply during the pandemics (EU Digital Solidarity: a call for a pan-European approach against 526 the pandemic, Wojciech Wiewiórowski, https://edps.europa.eu/sites/edp/files/publication 527 /2020-04-06_eu_digital_solidarity_covid19_en.pdf, 2020). Particular in a crisis situation such 528 as the current pandemic, Al systems need to comply with ethical principles and respect human 529 rights ${ }^{14}$. We therefore argue that systems such as Swarm Learning that allow fair, transparent 530 and still highly regulated shared data analytics while preserving data privacy regulations are 531 to be favored, particularly during times of high urgency to develop supportive tools for medical 532 decision making. We therefore also propose to explore SL for image-based diagnostics of 533 COVID-19 from patterns in X-ray images or computed tomography (CT) scans ${ }^{21,22}$, structured 534 health records ${ }^{67}$, or wearables for disease tracking ${ }^{14}$. Swarm learning would also have the 535 advantage that model and code sharing as well as dissemination of new applications is easily 536 scalable, because onboarding of new swarm participants is structured by blockchain 537 technology, while scaling of data sharing is not even necessary due the inherent local 538 computing of the data ${ }^{14}$. Furthermore, swarm learning can reduce the burden of establishing 539 global, comprehensive, open, and verified datasets.

540 Collectively, we introduce Swarm Learning defined by the combination of blockchain 541 technology and decentralized machine learning in an entirely democratized approach 
542 eliminating a central player and therefore representing a uniquely fitting strategy for the

543 inherently locally organized domain of medicine. We used blood transcriptomes in three

544 scenarios as use cases since they combine blood as the most widely used surrogate tissue

545 for diagnostic purposes with an omics technology producing high-dimensional data with many

546 parameters. Since the deployment of Swarm Learning due to ease of use of Swarm Learning

547 libraries is a rather simple task, we propose to expand the use of this technology and further

548 develop such classifiers in a unifying fashion across centers worldwide without any need to

549 share the data itself. Our use cases are supposed to serve as examples for other high-

550 dimensional data in the domain of medicine, but certainly also many other areas of research

551 and application against the pandemics and beyond. 
552

553

554

555

556

557

558

559

560

561

562

563

564

565

566

567

568

569

570

571 Funding:

572

This work was supported in part by the German Research Foundation (DFG) to J.L.S. (INST

573

574

575

576

577

578

579

580

581

582

\section{Acknowledgments:}

We thank Dr. Sach Mukherjee (Statistics and Machine Learning, German Center for Neurodegenerative Diseases) for feedback on the manuscript.

\section{Deutsche COVID-19 Omics Initiative (DeCOI)}

Robert Bals, Alexander Bartholomäus, Anke Becker, Ezio Bonifacio, Peer Bork, Thomas Clavel, Maria Colome-Tatche, Andreas Diefenbach, Alexander Dilthey, Nicole Fischer, Konrad Förstner, Julien Gagneur, Alexander Goesmann, Torsten Hain, Michael Hummel, Stefan Janssen, René Kallies, Birte Kehr, Andreas Keller, Sarah Kim-Hellmuth, Christoph Klein, Oliver Kohlbacher, Jan Korbel, Ingo Kurth, Markus Landthaler, Yang Li, Kerstin Ludwig, Oliwia Makarewicz, Manja Marz, Alice McHardy, Christian Mertes, Markus Nöthen, Peter Nürnberg, Uwe Ohler, Stephan Ossowski, Jörg Overmann, Klaus Pfeffer, Alfred Pühler, Nikolaus Rajewsky, Markus Ralser, Olaf Rieß, Stephan Ripke, Ulisses Nunes da Rocha, Philip Rosenstiel, Antoine-Emmanuel Saliba, Leif Erik Sander, Birgit Sawitzki, Philipp Schiffer, Joachim L. Schultze, Alexander Sczyrba, Oliver Stegle, Jens Stoye, Fabian Theis, Janne Vehreschild, Jörg Vogel, Max von Kleist, Andreas Walker, Jörn Walter, Dagmar Wieczorek, John Ziebuhr 37/1049-1, INST 216/981-1, INST 257/605-1, INST 269/768-1 and INST 217/988-1, INST 217/577-1); the HGF grant sparse2big, the EU projects SYSCID (grant number 733100) and ImmunoSep, and by HPE to the DZNE for generating whole blood transcriptome data from patients with COVID-19. Sofia Ktena is supported by the Hellenic Institute for the Study of Sepsis. The clinical study in Greece was supported by the Hellenic Institute for the Study of Sepsis. E.J.G.-B. received funding from the FrameWork 7 program HemoSpec (granted to the National and Kapodistrian University of Athens), the Horizon2020 Marie-Curie Project European Sepsis Academy (granted to the National and Kapodistrian University of Athens), and the Horizon 2020 European Grant ImmunoSep (granted to the Hellenic Institute for the Study of Sepsis). 


\section{Author contributions:}

584 Conceptualization, J.L.S; K.L.S, S.Ma.; Methodology, S.W.-H., C.S., R.S., M.D., B.M., C.M.S.; 585 Software: M.L.; V.G, C.S., S.Ma., S.Mu.; Investigation, S.W.-H., K.S., M.K., A.D., M.N., H.T., 586 T.U.; Biospecimen/ enzyme resources, M.K., P.P., M.G.N., S.K., E.G.-B., M.M.B.BM., C.K., 587 M.G.; Writing - Original Draft, S.W.-H., H.S., K.L.S., M.B., J.L.S.; Writing - Review \& Editing, 588 S.W.-H., H.S., K.L.S., D.S., A.C.A., M.K., P.P., M.G.N., M.B., S.C., M.S.W., E.L.G., J.L.S.; 589 Visualization, H.S., J.L.S; Supervision, H.S., K.L.S., J.L.S.; Project Administration, H.S., 590 J.L.S.; Funding Acquisition, W.F., J.L.S., F.J.T., J.H., N.Y., A.K.S., M.G., and M.M.B.B.

\section{Ethics declarations}

\section{Competing Interests}

595 H.S., K.L.S, S.Ma., S.Mu., V.G., R.S., C.S., M.D., B.M, C.M.S., S.C., M.S.W, E.L.G are 596 employees of Hewlett Packard Enterprise. Hewlett Packard Enterprise developed the Swarm 597 Learning Library in its entirety as described in this work and has submitted multiple associated 598 patent applications. E.J.G.-B. received honoraria from AbbVie USA, Abbott $\mathrm{CH}$, InflaRx $599 \mathrm{GmbH}, \mathrm{MSD}$ Greece, XBiotech Inc. and Angelini Italy; independent educational grants from 600 AbbVie, Abbott, Astellas Pharma Europe, AxisShield, bioMérieux Inc, InflaRx $\mathrm{GmbH}$, and 601 XBiotech Inc. 
bioRxiv preprint doi: https://doi.org/10.1101/2020.06.25.171009; this version posted June 29, 2020. The copyright holder for this preprint (which was not certified by peer review) is the author/funder, who has granted bioRxiv a license to display the preprint in perpetuity. It is made available under aCC-BY-NC-ND 4.0 International license.

\section{Figures and Figure Legends}

603

Figure 1
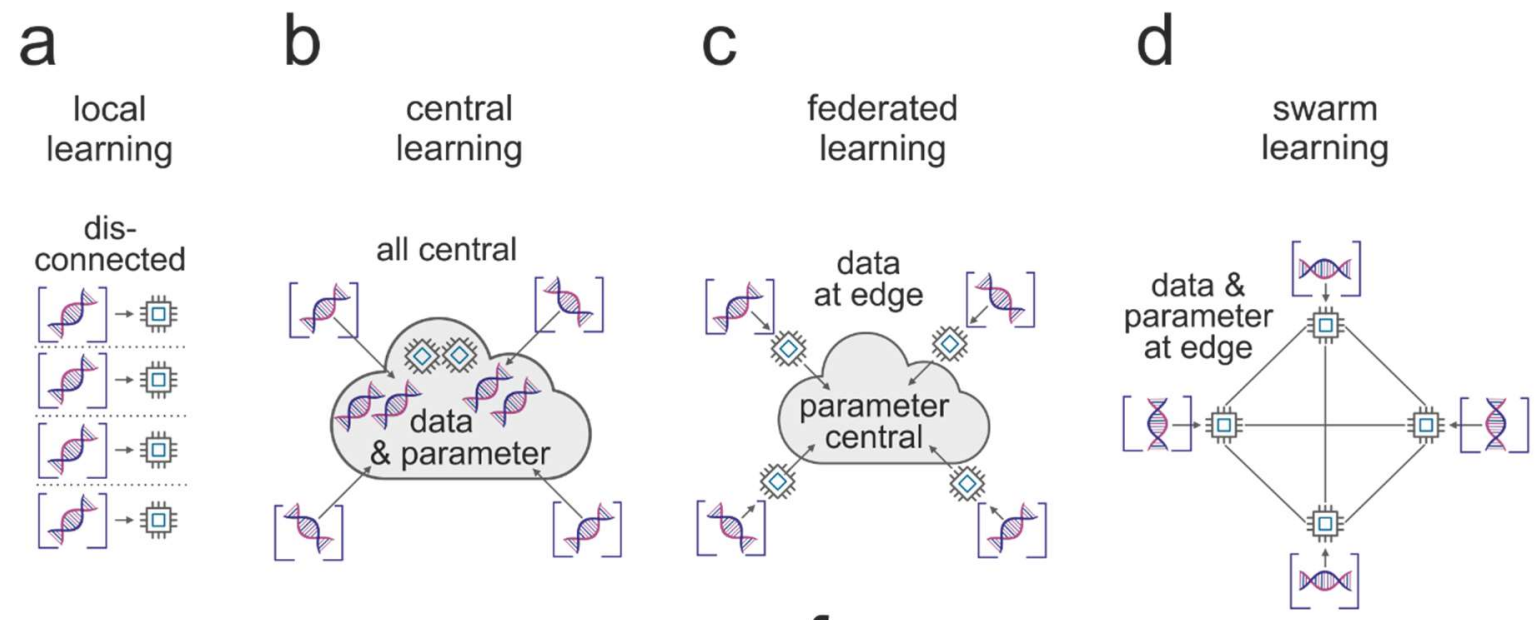

e
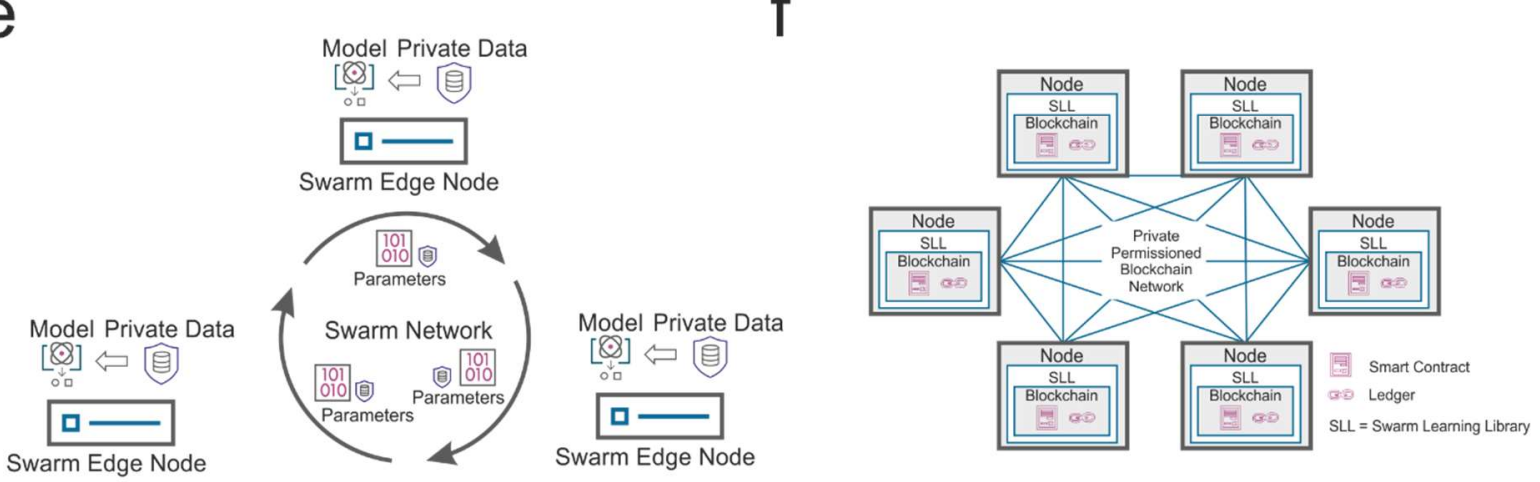

g

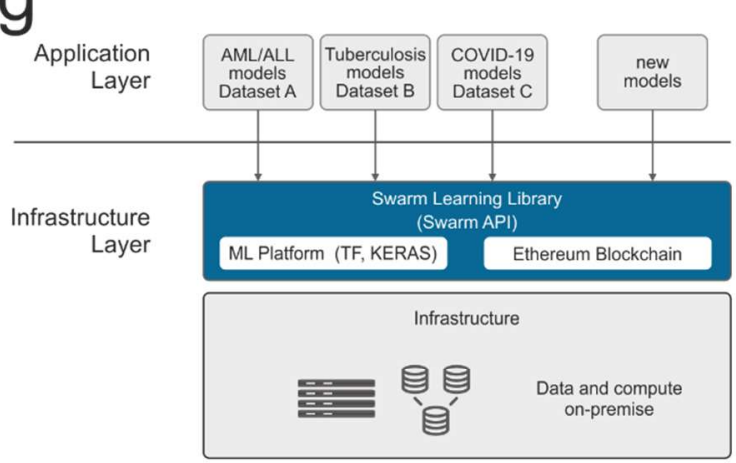

604 k

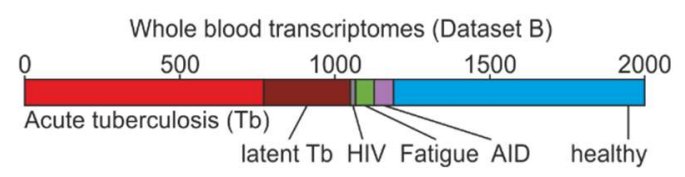

h
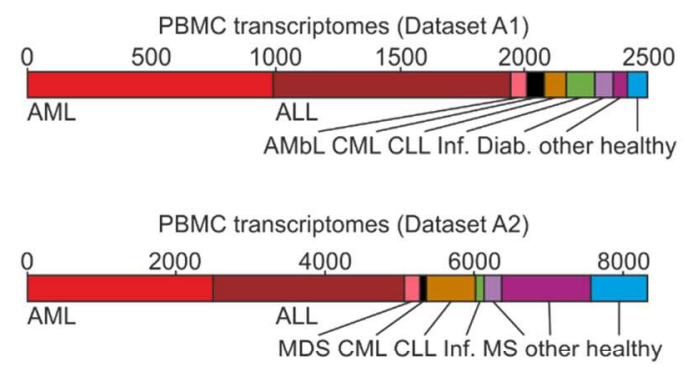

PBMC transcriptomes (Dataset A3)

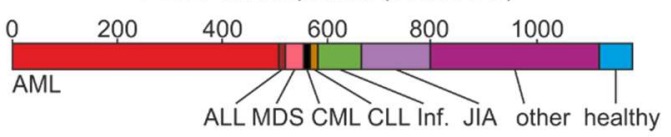

Whole blood transcriptomes (Dataset C)

COVID-19


607 (a-d) The principles of Swarm Learning in contrast to other machine learning concepts. (a) 608 Illustration of the concept of local learning with data and computation at different, but 609 disconnected locations. (b) Principle of cloud-based machine learning where data from 610 contributing centers move copies of the data to a central cloud-based storage; centrally 611 located data are then used for central - often cloud-based - machine learning. (c) Federated 612 learning with data being kept with the data contributor and computing is also performed at the 613 site of local data storage and availability, yet parameter settings of machine learning are 614 orchestrated by a central parameter server. (d) Swarm Learning principle with swarm nodes 615 being connected in a democratic fashion (enabled by blockchain technology) without the need 616 for a central custodian or parameter server. Data privacy is preserved, data is kept where it is 617 generated, computation is achieved locally and learning parameters are shared within the 618 Swarm Network. (e) Schematic representation of the Swarm Network consisting of the Swarm 619 Edge Nodes (short 'nodes') that exchange parameters for learning, which is implemented 620 using blockchain technology. Use of private data at each node together with the model 621 provided via Swarm Network. (f) Concept and outline of the private permissioned blockchain 622 network as a layer of the Swarm Learning network. Each node consists of the blockchain, 623 including the ledger and smart contract, as well as the Swarm Learning Library (SLL) with the 624 API to interact with other nodes within the network. (g) Application and infrastructure layer as 625 part of the Swarm Learning concept. (h-l) Description of the transcriptome datasets used 626 within this study: Dataset (h) A1 and (i) A2, two microarray-based transcriptome datasets of 627 peripheral blood mononuclear cells (PBMC). (j) Dataset A3, RNA-seq based transcriptomes 628 of PBMC. Dataset (k) B and (I) C, RNA-seq based whole blood transcriptome datasets. 629 Abbreviations: AML, Acute Myeloid Leukemia; ALL, Acute Lymphoblastic Leukemia; COVID630 19, CoronaVirus Disease 2019; API, Application Programming Interface; ML, Machine 631 Learning; TF, Tensor Flow; KERAS, Open Source Deep Learning Library; AMbl, Acute 632 Myeloblastic Leukemia; CML, Chronic Myeloid Leukemia; CLL, Chronic Lymphocytic 633 Leukemia; Inf., Infections, Diab., Diabetes Type II; MDS, Myelodysplastic Syndrome; MS, 634 multiple sclerosis; JIA, Juvenile idiopathic arthritis; Tb, tuberculosis; HIV, Human 635 Immunodeficiency Virus, AID, Acute Infectious Disease. SLL Swarm Learning Library. 
bioRxiv preprint doi: https://doi.org/10.1101/2020.06.25.171009; this version posted June 29, 2020. The copyright holder for this preprint (which was not certified by peer review) is the author/funder, who has granted bioRxiv a license to display the preprint in perpetuity. It is made available under aCC-BY-NC-ND 4.0 International license.

\section{Figure 2}

a

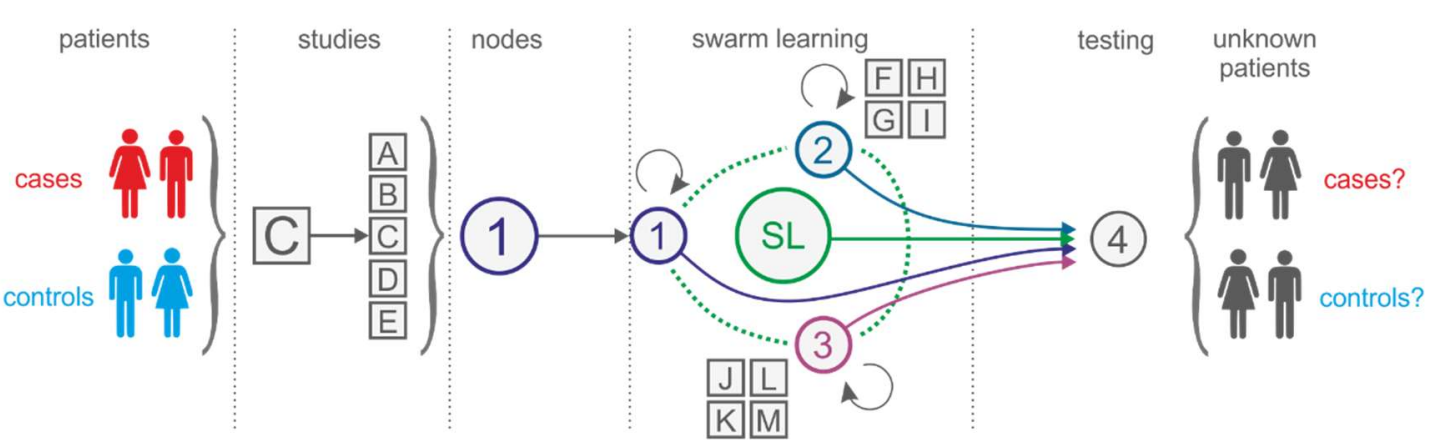

b

Dataset A2

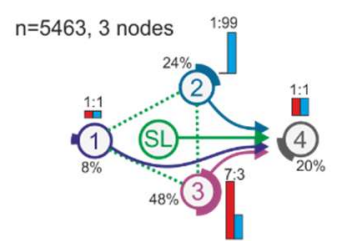

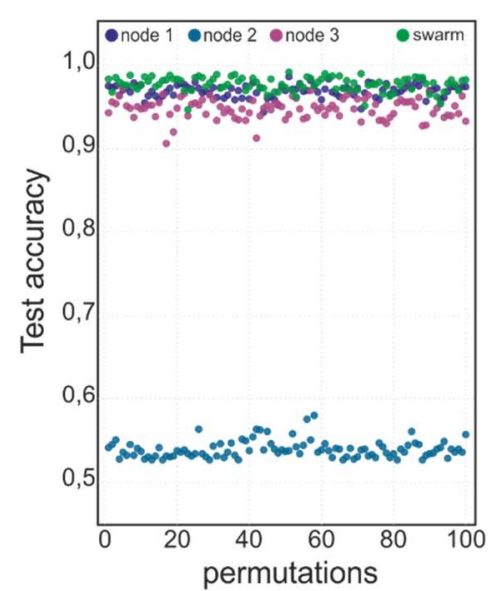

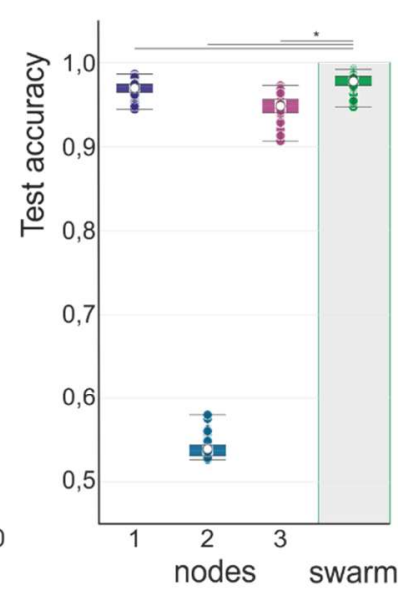

C
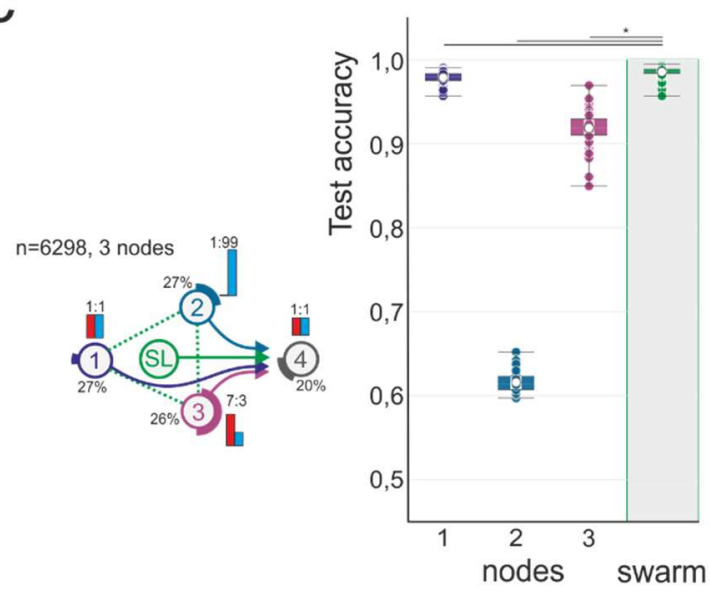

e
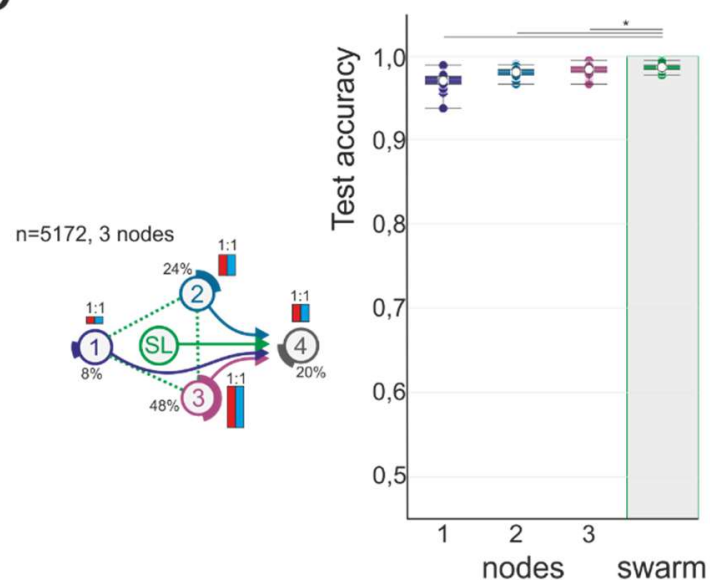

d

f
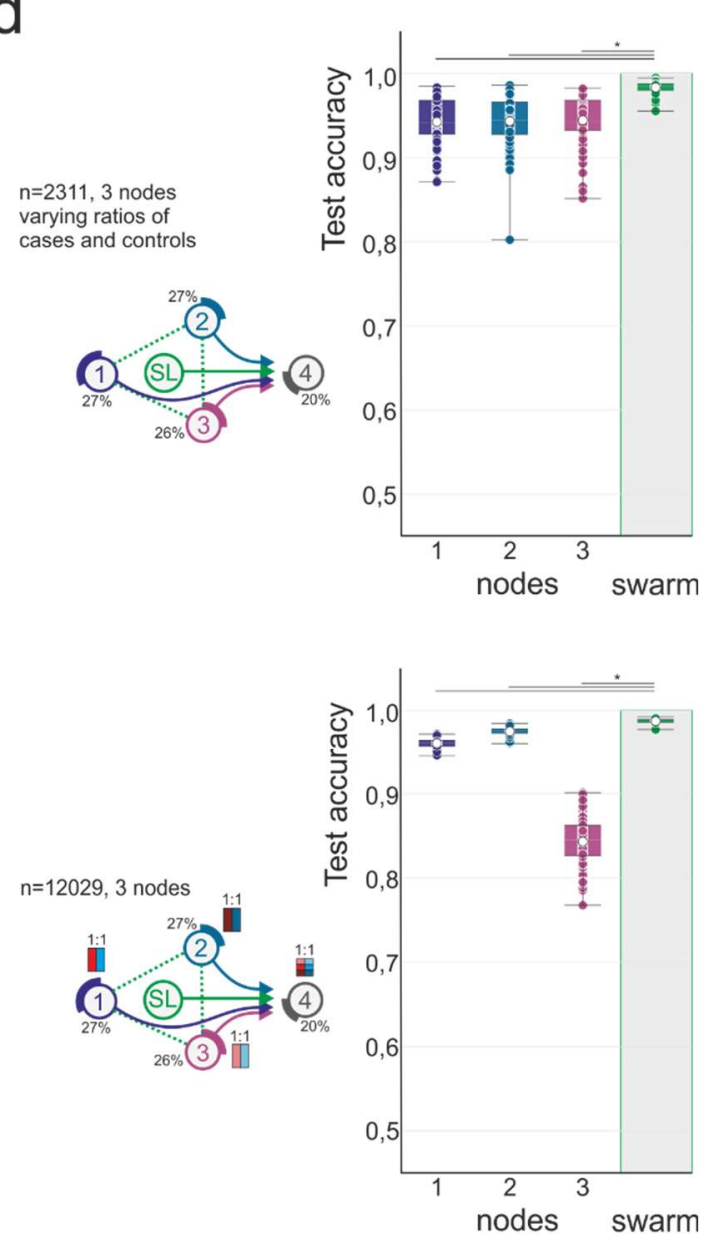


\section{Figure 2. Swarm learning to predict leukemias from PBMC data}

638 (a) Schematic representation of the use of the transcriptome data derived from more than 63912,000 individuals in over 100 individual studies ${ }^{47}$. Principle of distribution of data to individual

640 Swarm Edge Nodes (short 'nodes'). Nodes 1-3 were used for training, node 4 for testing.

641 Swarm Learning (SL) was achieved by integrating nodes 1-3 for training following procedures

642 described in detail in Supplementary Information. (b) Scenario using Dataset A2. Left panel

643 illustrating the setting of the scenario concerning distribution of cases and controls to individual

644 nodes, as well as total number of samples used for this scenario. Cases (red bar) and controls

645 (blue bar) were distributed unevenly among nodes, the number of samples distributed to each

646 node was also uneven in this scenario. Middle panel shows results of accuracy of all 100

647 permutations performed for the 3 training nodes individually as well as the results obtained by

648 SL. Accuracy is defined for the independent fourth node used for testing only. Right panel

649 represents box-whisker plot representation of the individual data presented in the middle panel

650 showing mean, 1st and 3rd quartile and whisker type Min/Max. (c) Scenario with uneven

651 numbers of cases and controls at the different training nodes but similar numbers of samples

652 at each node to determine impact of these changes on SL performance. Left panel: schematic

653 representation of scenario and right panel: results obtained for accuracy at the test node (node

654 4) for each of the three training nodes 1-3 and SL independently as box and whisker plot with

655 the same parameter as described for (b). (d) Scenario with even numbers at each of the

656 nodes, schematic representation (left panel) and visualization of results as box-whisker plots

657 as in (b) and (c). (e) Scenario with even distribution of cases and controls at each training

658 node, but different numbers of samples at each node and overall increase in numbers of 659 samples. Representation of schema and data visualization as in (b-d). (f) Scenario where each 660 node obtained samples from different Datasets (node 1: Dataset A1, node 2: Dataset A2, node 661 3, Dataset A3). Node 4 obtained samples from each Dataset A1-A3 to define impact on 662 technical bias on Swarm Learning performance. Representation of schema and data 663 visualization as in (b-e). Statistical differences between results derived by SL and individual 664 nodes including all permutations performed were calculated with Wilcoxon signed rank test 665 with continuity correction; asterisk and line: $p<0.05$. 
bioRxiv preprint doi: https://doi.org/10.1101/2020.06.25.171009; this version posted June 29, 2020. The copyright holder for this preprint (which was not certified by peer review) is the author/funder, who has granted bioRxiv a license to display the preprint in perpetuity. It is made available under aCC-BY-NC-ND 4.0 International license.

\section{Figure 3}

a

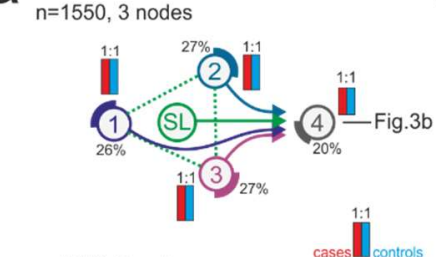

$C^{n=1550,6 \text { nodes }}$

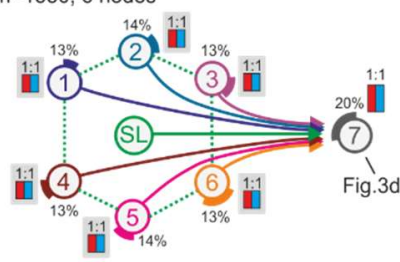

e<smiles>OC(O)C1OCCO1</smiles>

$\mathrm{g}_{\mathrm{n}=1300,3 \text { nodes }}$

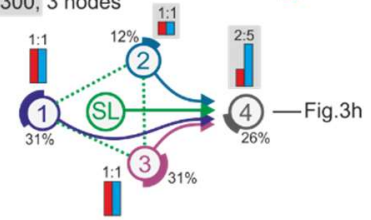

$\mathbf{i}_{n=850,3 \text { nodes }} \quad \mathbf{j}$

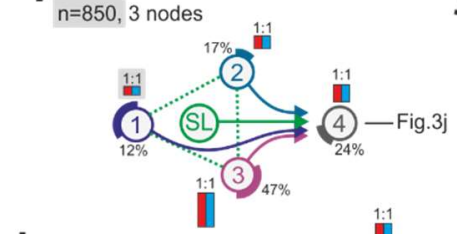

$K_{n=1000,3 \text { nodes }}$

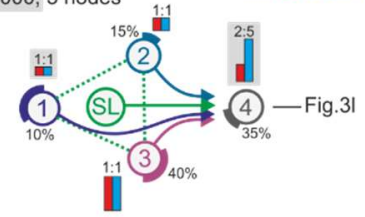

f b
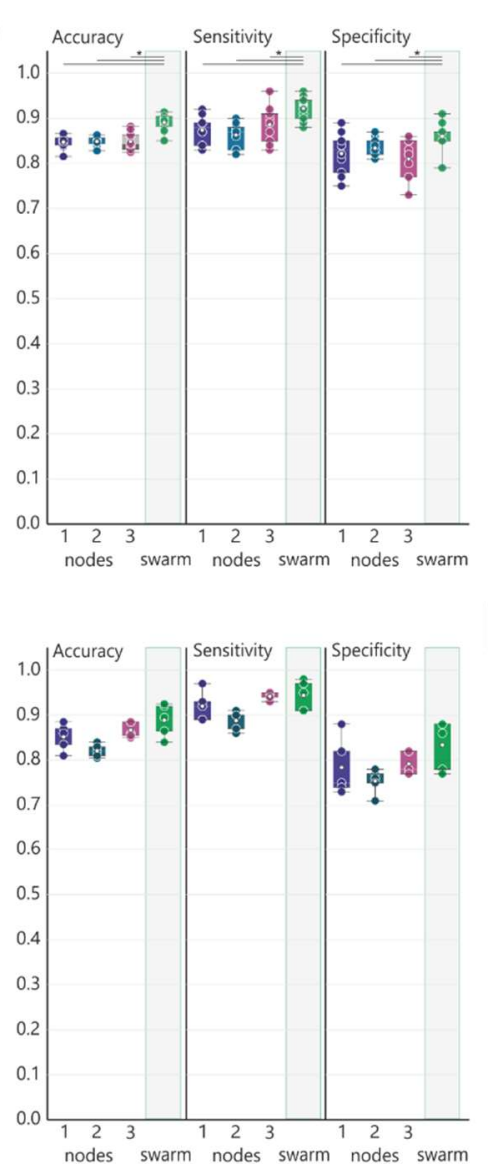

j

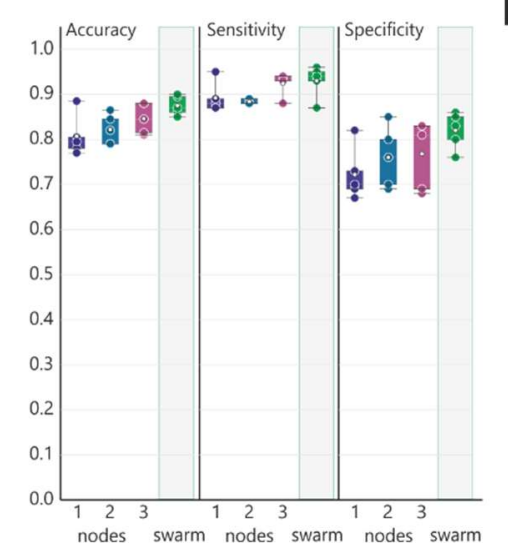

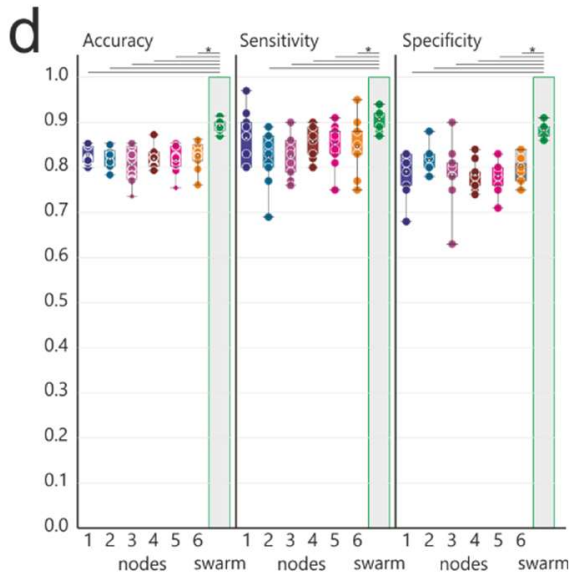

h
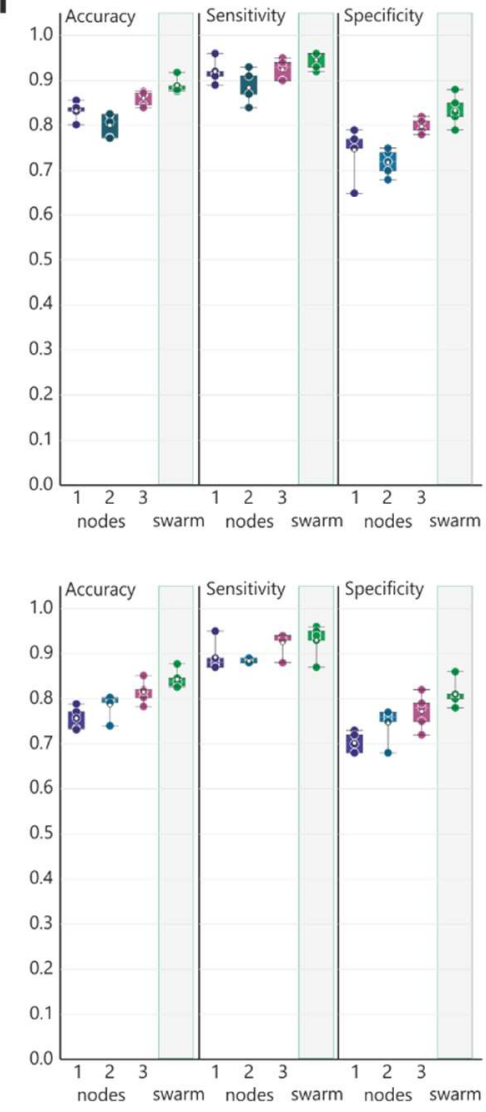


\section{Figure 3. Swarm learning to identify patients with tuberculosis}

669 (a-I) Principle of distribution of data to individual Swarm Edge Nodes (short 'nodes'). Nodes

670 1-3 were used for training, node 4 for testing. Swarm Learning (SL) was achieved by

671 integrating nodes 1-3 for training following procedures described in detail in Supplementary

672 Information. All scenarios use dataset B and use acute TB as case and the remaining samples

673 as controls. Left panels (a,c,e, $\mathbf{g}, \mathbf{i}, \mathbf{k})$ illustrate the setting of the scenarios concerning 674 distribution of cases (red bar) and controls (blue bar) to individual nodes, as well as total 675 number of samples used for the scenario. Percentage at each node reflects the use of samples 676 out of the complete dataset. (a) Scenario with even number of cases at each training node 677 and the test node. (b) Evaluation of the scenario presented in (a) showing accuracy, sensitivity 678 and specificity of five permutations for each training node and SL at node 4 (test node) as box679 whisker plot (mean, 1st and 3rd quartile, whisker type Min/Max). (c) Scenario similar to (a) but 680 with six training nodes. (d) Evaluation of scenario (c) as described in (b) but for all six training 681 nodes. (e) Scenario where the training nodes have evenly distributed numbers of cases and 682 controls at each training node, but node 2 has lower numbers of samples. (f) Evaluation of 683 scenario (e) as described in (b). (g) Scenario similar to (e) but with reduced prevalence at the 684 test node. (h) Evaluation of scenario (g) as described in (b). (i) Scenario with even distribution 685 of cases and controls at each training node, but node 1 only has a very small training set. The 686 test set is evenly distributed. (j) Evaluation of scenario (i) as described in (b). (k) Scenario 687 similar to (i) but with uneven distribution in the test node. (I) Evaluation of scenario (k) as 688 described in (b). Statistical differences between results derived by SL and individual nodes 689 including all permutations performed were calculated with Wilcoxon signed rank test with continuity correction; asterisk and line: $p<0.05$. 
bioRxiv preprint doi: https://doi.org/10.1101/2020.06.25.171009; this version posted June 29, 2020. The copyright holder for this preprint (which was not certified by peer review) is the author/funder, who has granted bioRxiv a license to display the preprint in perpetuity. It is made available under aCC-BY-NC-ND 4.0 International license.

\section{Figure 4}

a

$\mathrm{n}=899,3$ training nodes, 1 test node

different prevalences

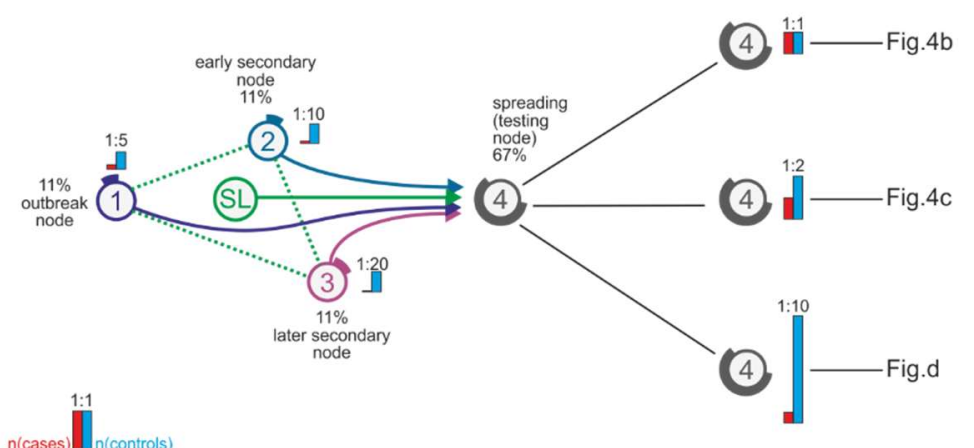

b

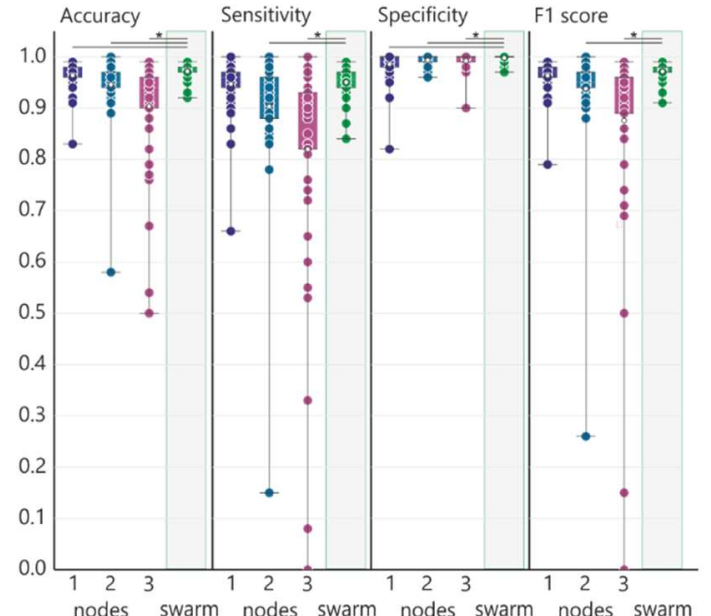

d

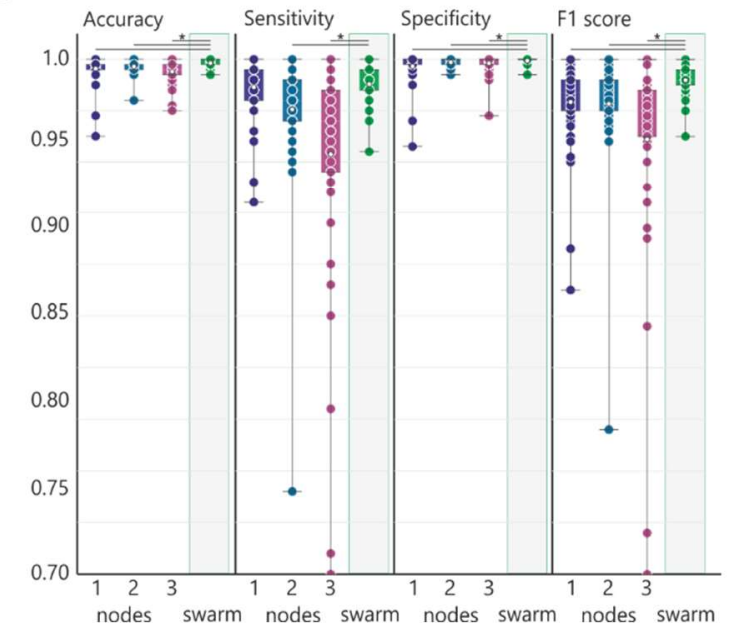

C

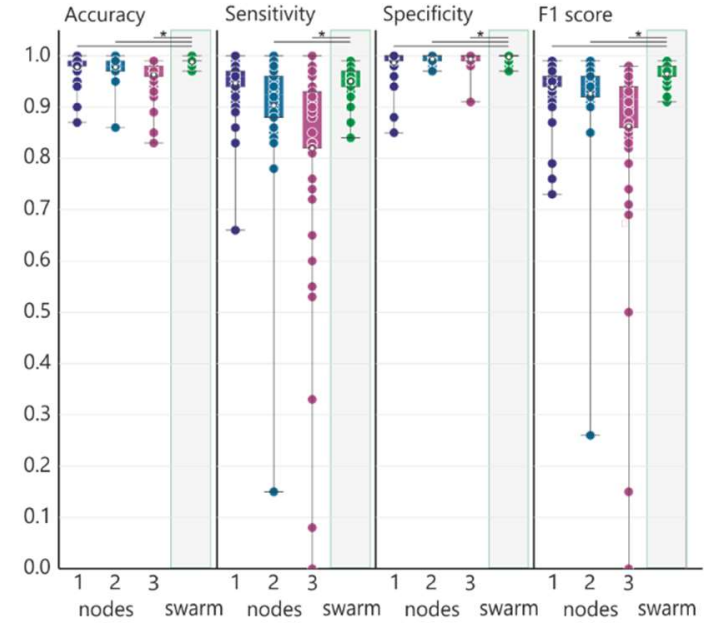




\section{Figure 4. Identification of COVID-19 patients in an outbreak scenario}

694 (a) Description of an outbreak scenario for COVID-19 using Dataset C. Nodes 1-3 were used

695 for training, node 4 for testing. Swarm Learning (SL) was achieved by integrating nodes 1-3

696 for training following procedures described in detail in Supplementary Information. COVID-19

697 samples were used as cases. In this scenario, node 1 would be the outbreak node with the

698 highest prevalence. Training node 2 has fewer cases and is an early secondary node, and

699 node 3 acts as a later secondary node. The spreading is tested on the testing node with three

700 different prevalences (b,c,d) and shown as box-whisker plot (mean, 1st and 3rd quartile,

701 whisker type Min/Max). (b) Evaluation of (a) with even prevalence showing accuracy,

702 sensitivity, specificity and F1-score of fifty permutations for each training node and the SL

703 (node 4). (c) Evaluation (as described in (b)) of scenario (a) using a 1:2 ratio for cases and

704 controls in the test set. (d) Evaluation (as described in (b)) of scenario (a) using a 1:10 ratio in

705 the test set to simulate detection in regions with new infections. Statistical differences between

706 results derived by SL and individual nodes including all permutations performed were

707 calculated with Wilcoxon signed rank test with continuity correction; asterisk and line: $p<0.05$. 


\section{Extended Data Figure 1}

a

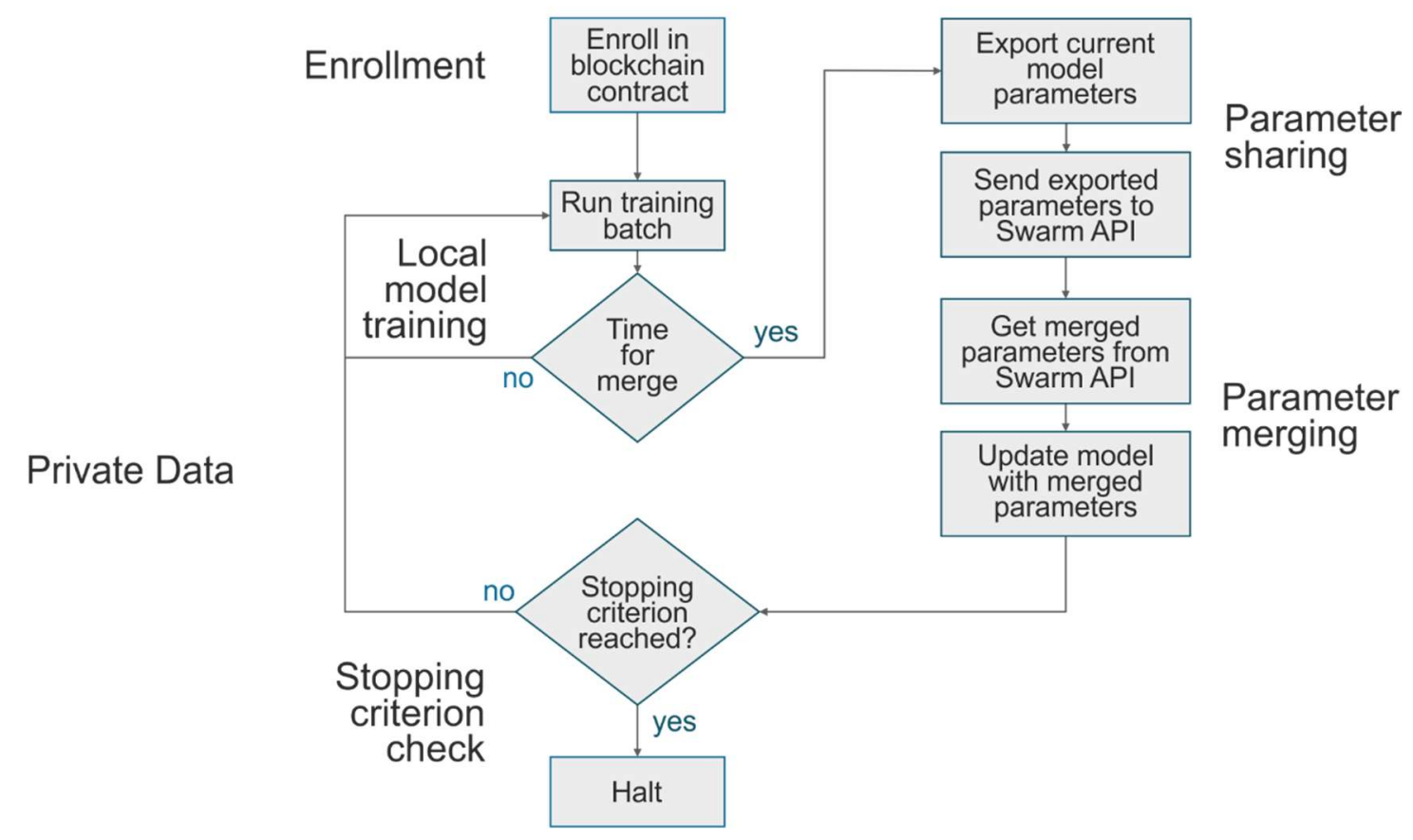

710

711 Extended Data Figure 1. corresponding to Fig. 1

712 Schematics of the principles of the workflow of Swarm Learning once the nodes have been

713 enrolled within the Swarm Network via private permissioned blockchain contract. 


\section{Extended Data Figure 2}

a

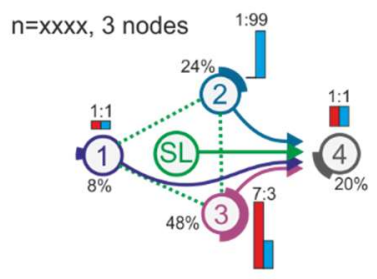

b
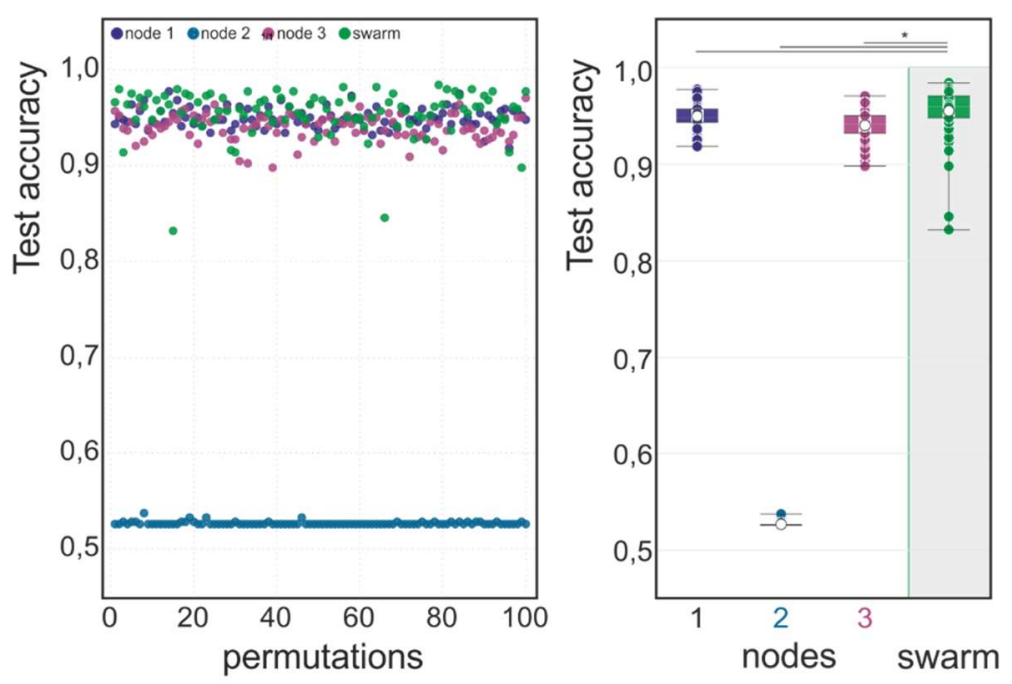

dataset $\mathrm{A} 1$

C
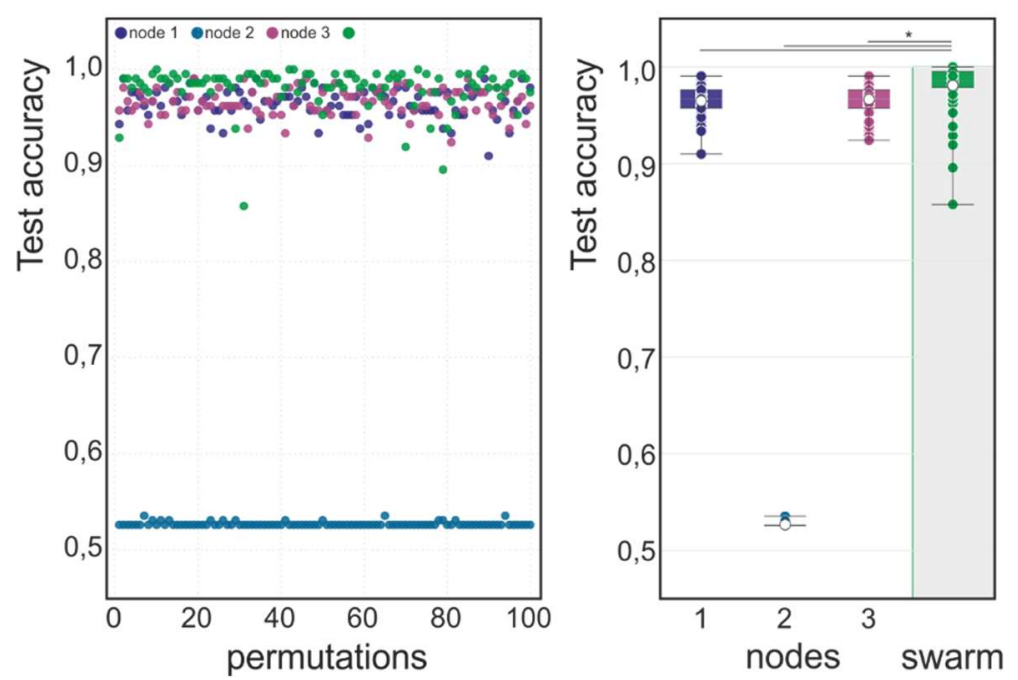

dataset $\mathrm{A} 3$ 


\section{Extended Data Figure 2. Scenario corresponding to Fig. 2b in dataset A1 and A3}

717 Main settings are identical to what is described in Fig. 2 for Dataset A2. (a) Scenario with 718 different prevalence of $A M L$ and different number of samples at each training node. The test 719 set has an even distribution. (b) Evaluation of test accuracy for 100 permutations of dataset

720 A1 per node and swarm. (c) Evaluation using dataset A3. Statistical differences between

721 results derived by $\mathrm{SL}$ and individual nodes including all permutations performed were 722 calculated with Wilcoxon signed rank test with continuity correction; asterisk and line: $p<0.05$. 


\section{Extended Data Figure 3}

\section{a}

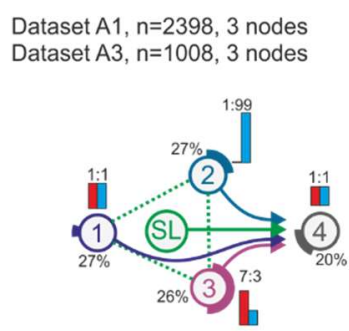

C

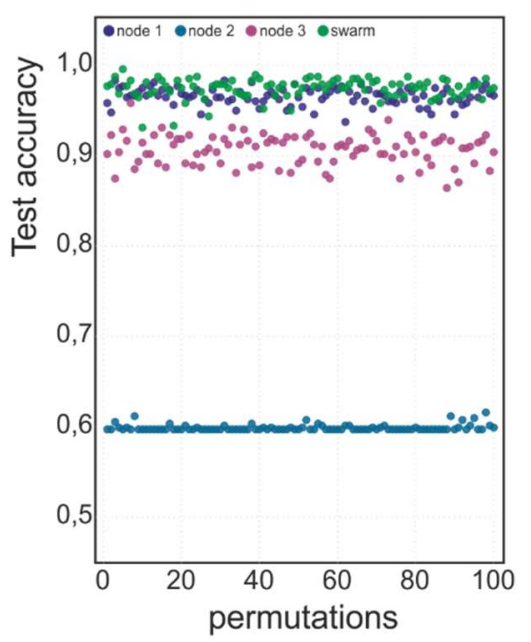

d

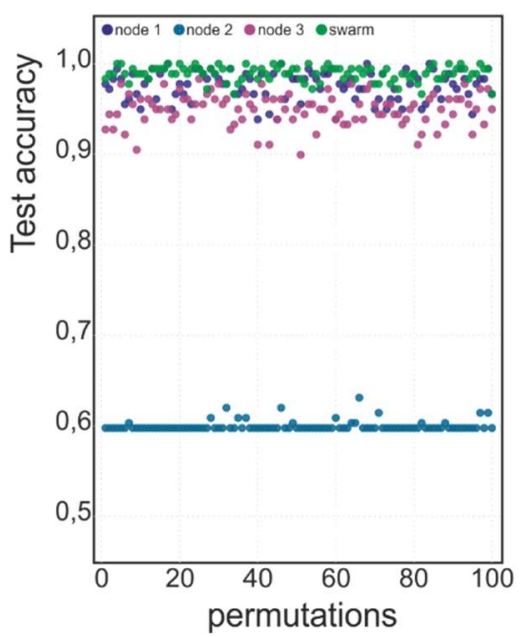

b

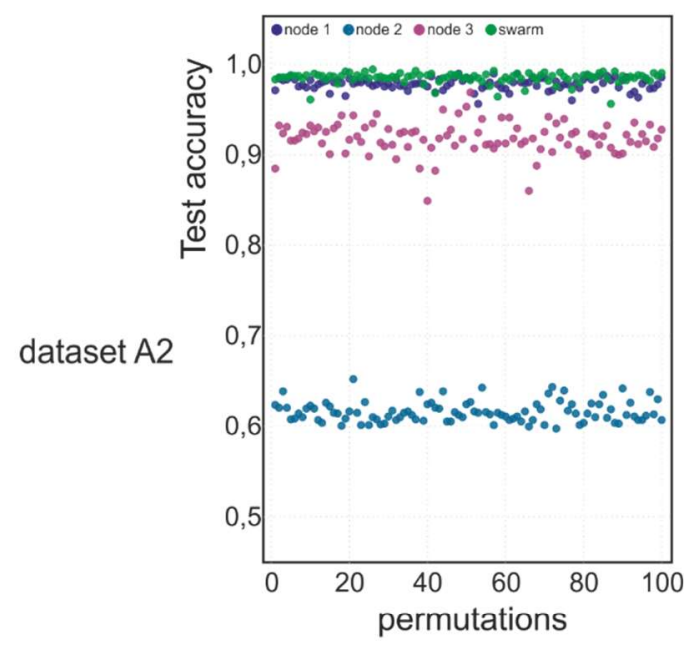

dataset A1

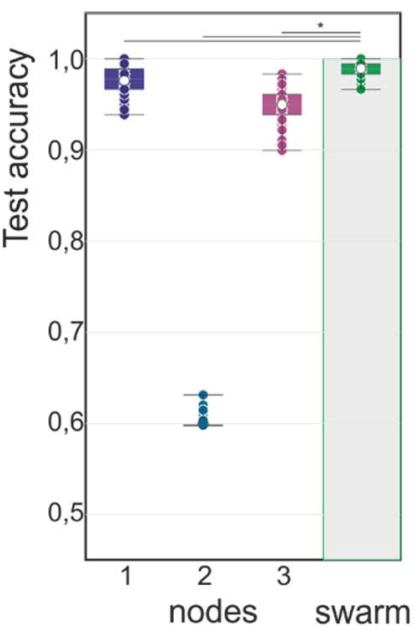

dataset $\mathrm{A} 3$ 


\section{Extended Data Figure 3. Scenario corresponding to Fig. 2c in dataset A1 and A3}

725 Main settings are identical to what is described in Fig. 2 for dataset A2. (a) Scenario with 726 similar training set sizes per node but decreasing prevalence. The test set ratio is 1:1. (b) 727 Evaluation of the test accuracy over 100 permutation for dataset A2 (corresponding to Fig.

728 2c). (c) Evaluation of the test accuracy over 100 permutation for dataset $A 1$. (d) Evaluation of 729 the test accuracy over 100 permutation for dataset A3. Box-whisker plots (mean, 1st and 3rd 730 quartile, whisker type Min/Max). Statistical differences between results derived by SL and 731 individual nodes including all permutations performed were calculated with Wilcoxon signed 732 rank test with continuity correction; asterisk and line: $p<0.05$. 


\section{Extended Data Figure 4}

a

Dataset $A 1, n=2311,3$ nodes

Dataset $A 3, n=1083$, 3 nodes

varying ratios of cases and controls

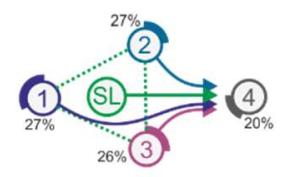

C

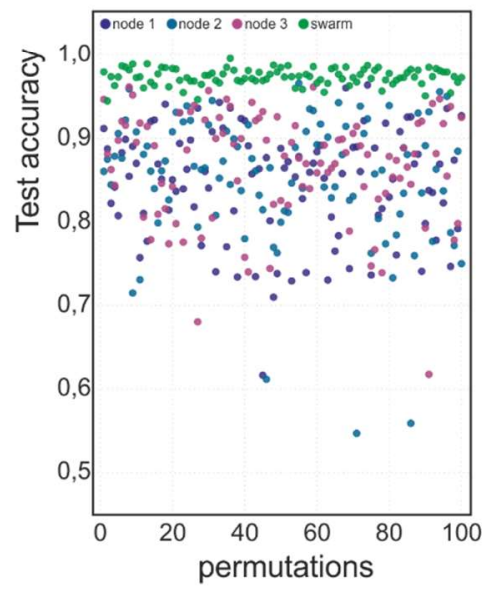

d

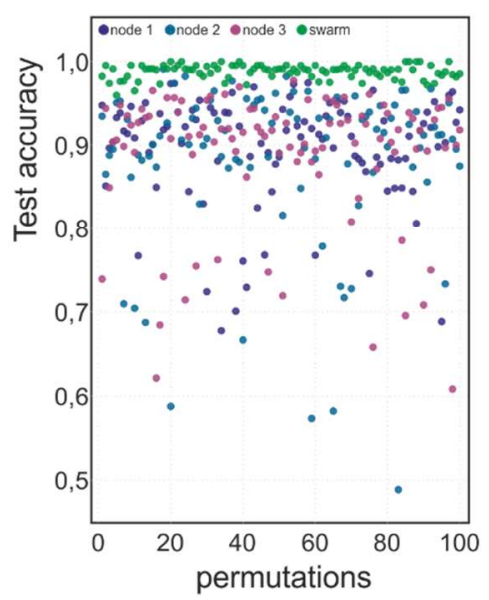

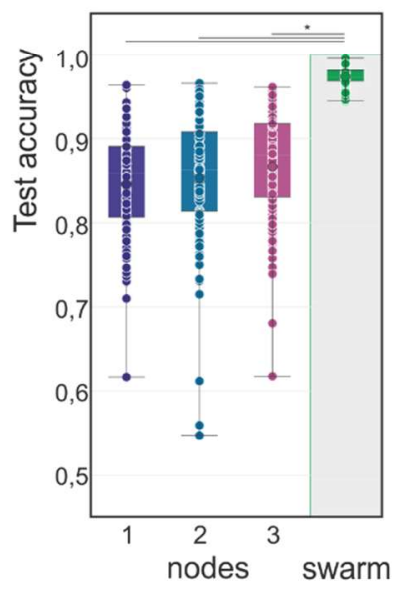

dataset $\mathrm{A} 2$

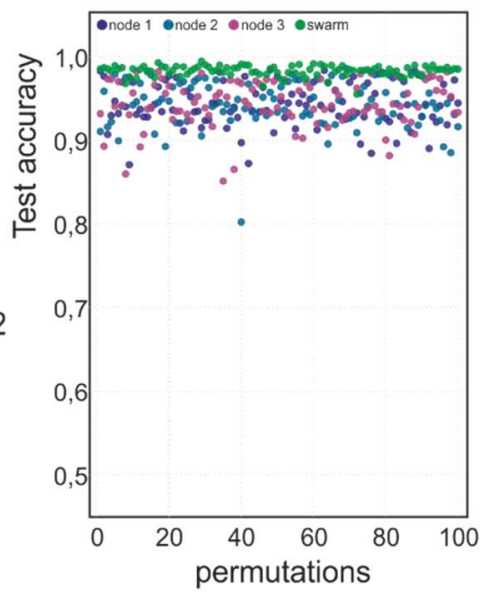

dataset A1
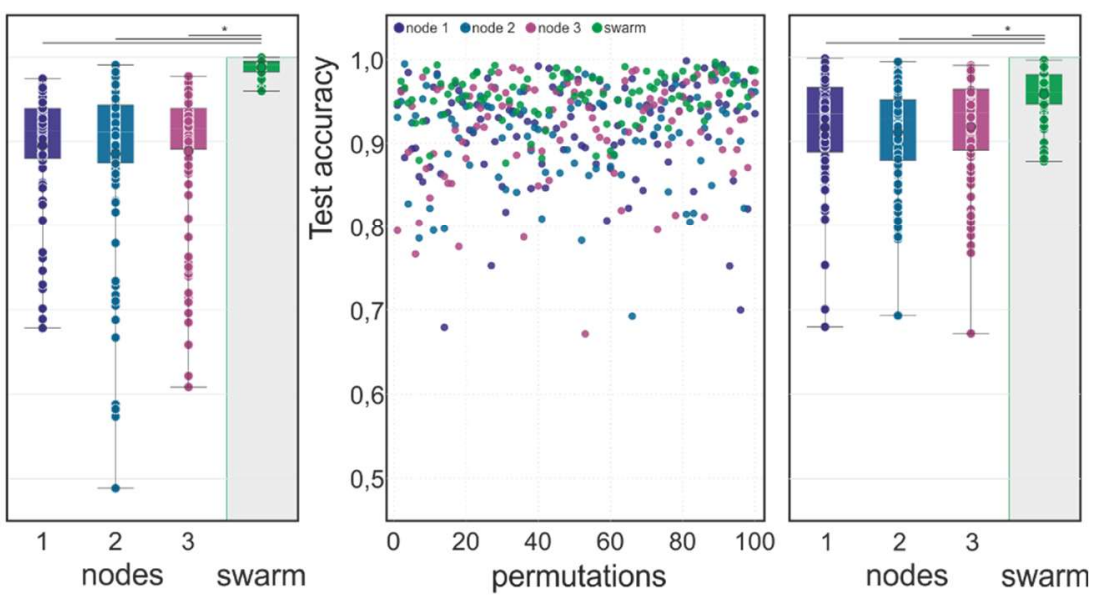

dataset $\mathrm{A} 3$

dataset A2 


\section{Extended Data Figure 4. Scenario corresponding to Fig. 2d in dataset A1 and A3}

736 Main settings are identical to what is described in Fig. 2 for dataset A2. (a) Scenario with

737 similar sample sizes among three nodes, but with independent studies at each training node.

738 Case and control ratios varied for each permutation. Testing samples are sampled from the

739 studies also present in the training data. (b) Evaluation of the test accuracy over 100

740 permutation for dataset A2 (corresponding to Fig. 2d). (c) Evaluation of the test accuracy over

741100 permutation for dataset A1. (d) Evaluation of the test accuracy over 100 permutation for

742 dataset A3. (e) In this scenario, samples at the test node were derived from published studies

743 completely independent from the studies used for training at the training nodes. Evaluation of

744 the test accuracy over 100 permutation for dataset A2. Box-whisker plots (mean, 1st and 3rd

745 quartile, whisker type Min/Max). Statistical differences between results derived by SL and

746 individual nodes including all permutations performed were calculated with Wilcoxon signed

747 rank test with continuity correction; asterisk and line: $p<0.05$. 


\section{Extended Data Figure 5}

\section{a \\ b}
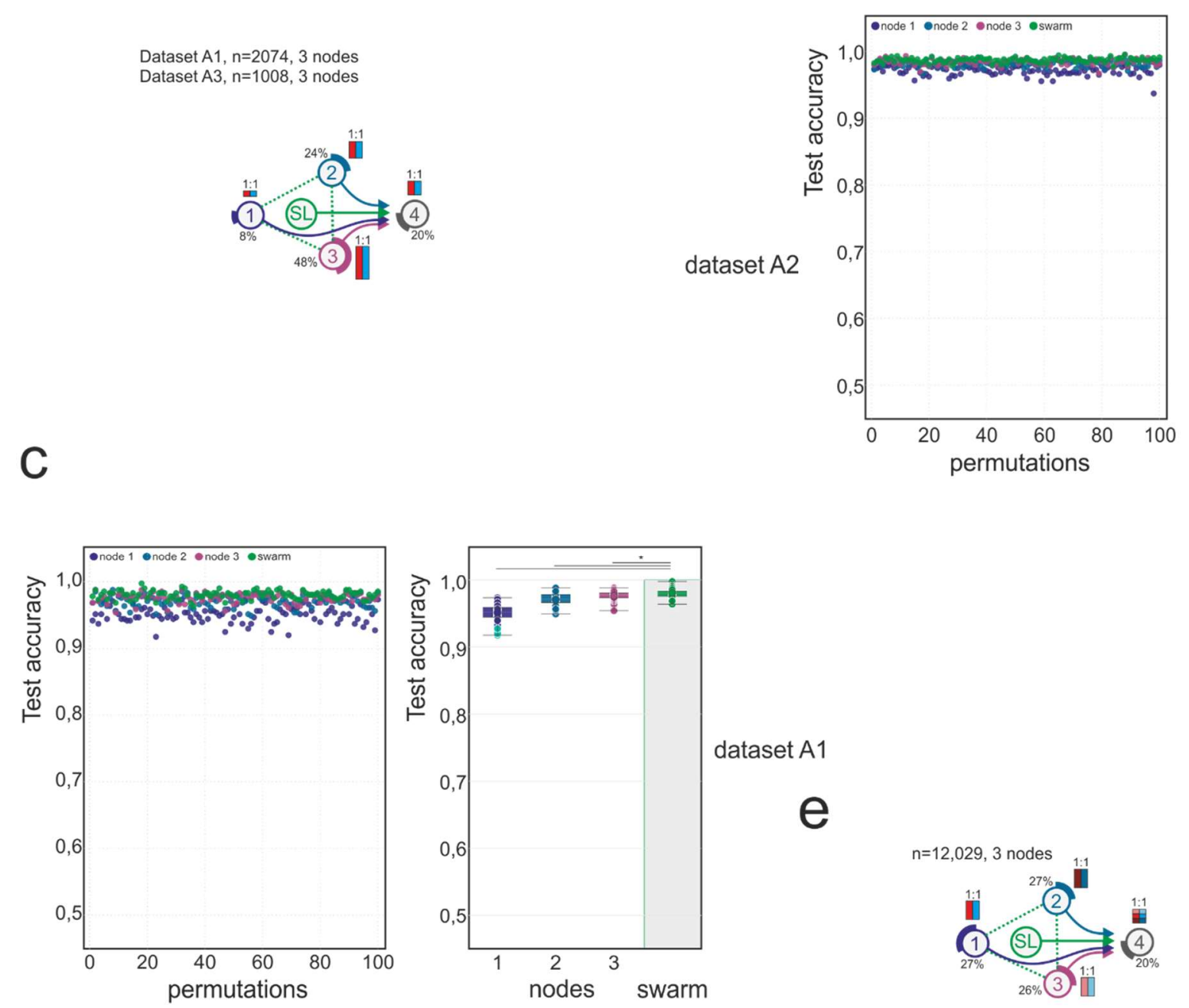

dataset $\mathrm{A} 1$

e

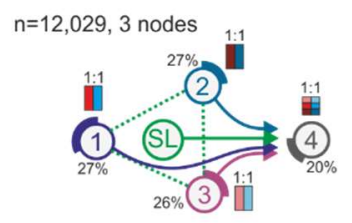

d
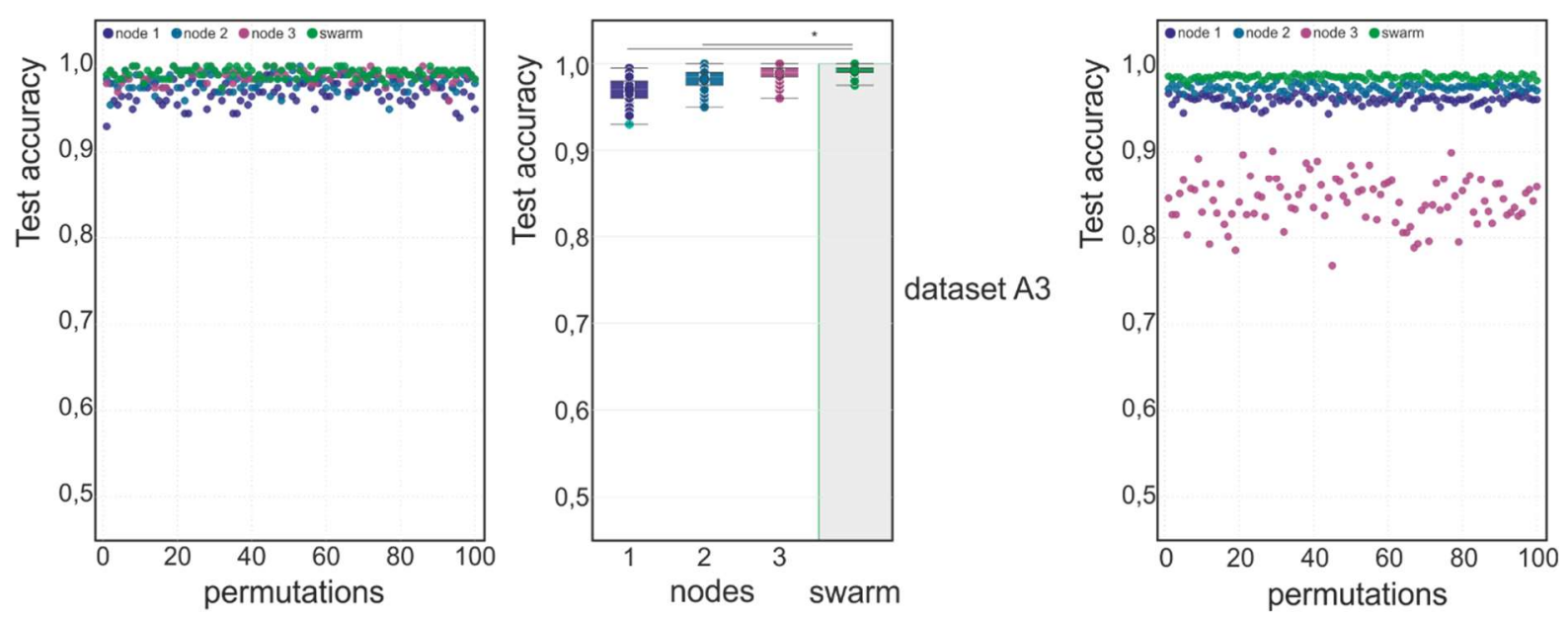


\section{Extended Data Figure 5. Scenario corresponding to Fig. $2 e$ in dataset A1 and A3}

751 Main settings are identical to what is described in Fig. 2 for dataset A2. (a) The case:control

752 distribution is even, the training sets increase from node 1 to node 3 . The test set is evenly

753 split. (b) Test accuracy for evaluation of dataset A2 (corresponding to Fig. 2e). (c) Test

754 accuracy for evaluation of dataset A1. (d) Test accuracy for evaluation of dataset A3. (e)

755 Scenario where the data sets $A 1, A 2$, and $A 3$ are assigned to a single training node each.

756 Scenario similar to (a) but with equal training set sizes. (f) Evaluation results of 100

757 permutations (corresponding to Fig. 2f). Box-whisker plots (mean, 1st and 3rd quartile, whisker

758 type Min/Max). Statistical differences between results derived by SL and individual nodes

759 including all permutations performed were calculated with Wilcoxon signed rank test with

760 continuity correction; asterisk and line: $p<0.05$. 
bioRxiv preprint doi: https://doi.org/10.1101/2020.06.25.171009; this version posted June 29, 2020. The copyright holder for this preprint (which was not certified by peer review) is the author/funder, who has granted bioRxiv a license to display the preprint in perpetuity. It is made available under aCC-BY-NC-ND 4.0 International license.

\section{Extended Data Figure 6 \\ a}

Dataset $A 2, n=2318,3$ nodes

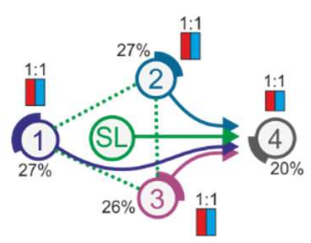

b
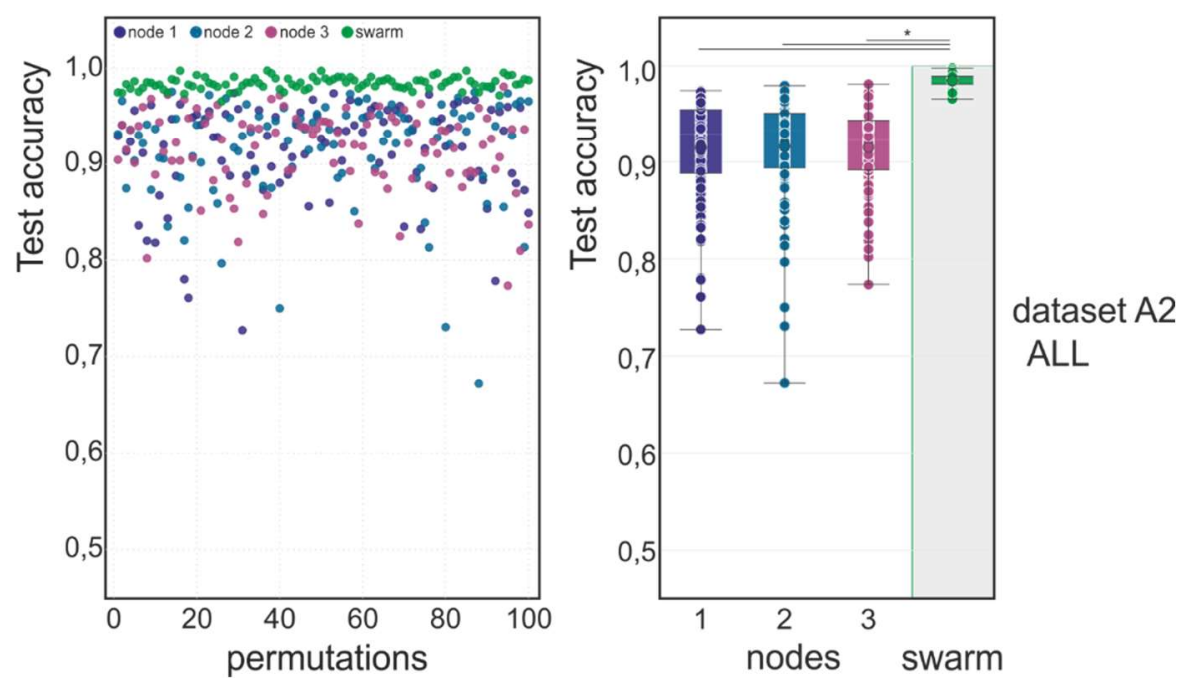

762 


\section{Extended Data Figure 6. Scenario for ALL in dataset 2}

764 Main settings are identical to what is described in Fig. 2 for dataset A2. Here cases are

765 samples derived from patients with ALL, while all other samples are controls (including AML).

766 (a) Scenario for the detection of ALL in dataset A2. The training sets are evenly distributed 767 among the nodes. The test ratio is 1:1. (b) Evaluation of scenario (a) for test accuracy over 768100 permutations. Box-whisker plot (mean, 1st and 3rd quartile, whisker type Min/Max). 769 Statistical differences between results derived by SL and individual nodes including all 770 permutations performed were calculated with Wilcoxon signed rank test with continuity 771 correction; asterisk and line: $p<0.05$. 
bioRxiv preprint doi: https://doi.org/10.1101/2020.06.25.171009; this version posted June 29, 2020. The copyright holder for this preprint

\section{Extended Data Figure 7}

\section{a}

group settings in use case 2 (tuberculosis)

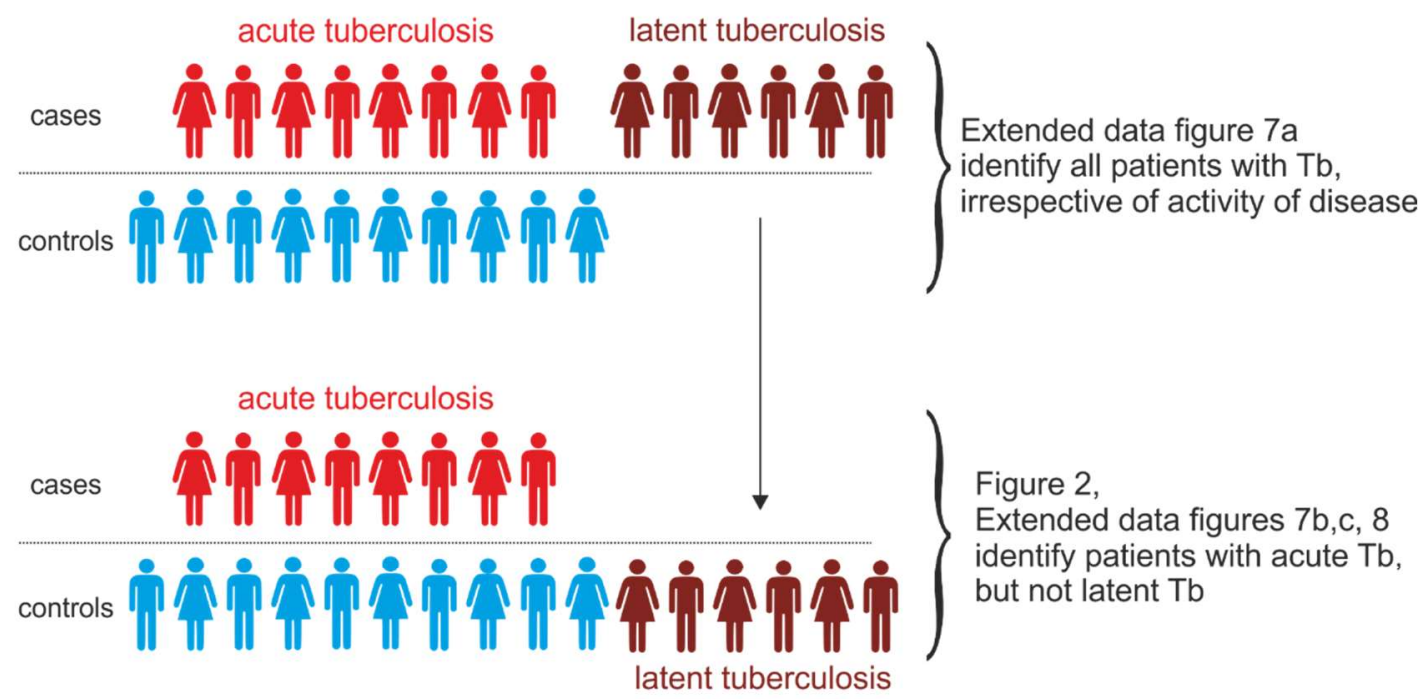

b<smiles>COc1ccco1</smiles>

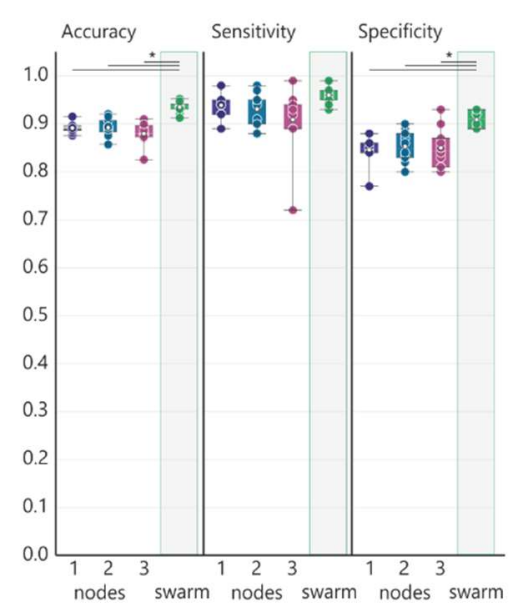

C

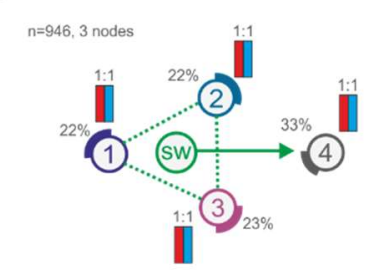

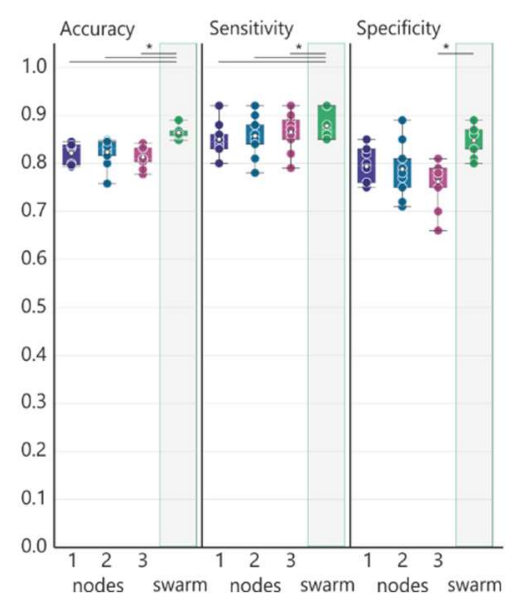

Only scenario for which the following applies: acute and latent TB are cases versus controls

\section{-

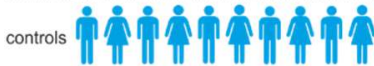

All other scenarios:

Only acute TB cases

versus

controls (now also including latent Tb cases) acute tuberculosis

cass

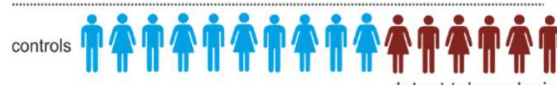




\section{Extended Data Figure 7. Scenario for detecting all Tb versus controls}

774 (a) Description of the different group settings used based on the assignment of latent Tb to 775 control or case. (b) Evaluation of a scenario where acute and latent Tb are cases. The data is 776 evenly distributed among the training nodes. The scenario is evaluated as described in Figure

7773 (b). (c) Scenario designed similar to (b) but latent Tb is part of control. Box-whisker plot 778 (mean, 1st and 3rd quartile, whisker type Min/Max). Statistical differences between results 779 derived by SL and individual nodes including all permutations performed were calculated with 780 Wilcoxon signed rank test with continuity correction; asterisk and line: $p<0.05$. 
bioRxiv preprint doi: https://doi.org/10.1101/2020.06.25.171009; this version posted June 29, 2020. The copyright holder for this preprint (which was not certified by peer review) is the author/funder, who has granted bioRxiv a license to display the preprint in perpetuity. It is made available under aCC-BY-NC-ND 4.0 International license.

\section{Extended Data Figure 8}

a

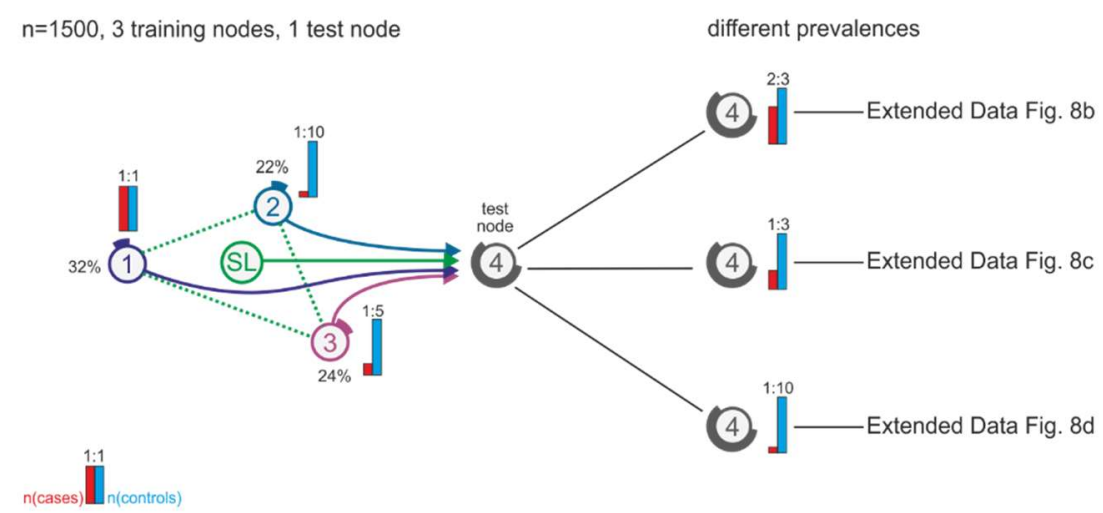

b

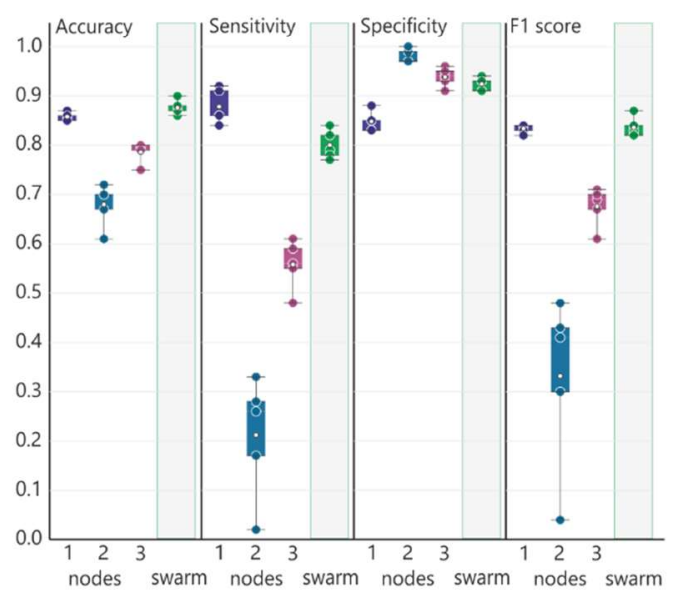

d

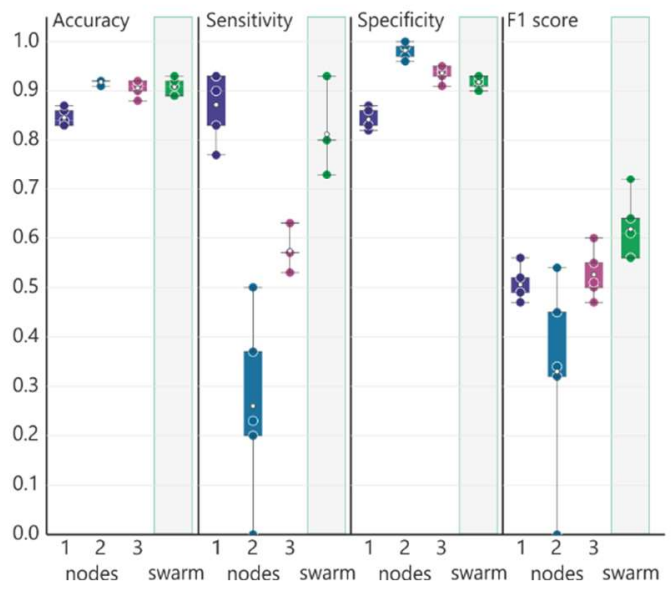

C

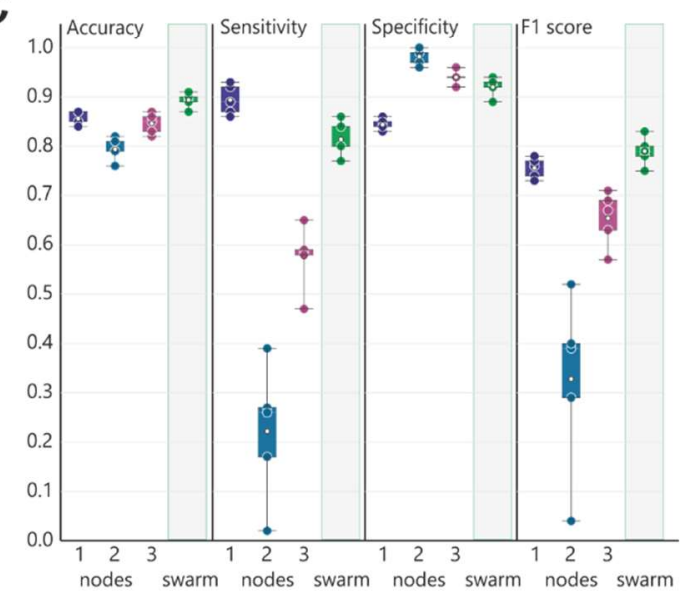




\section{Extended Data Figure 8: Scenario detecting acute Tb with low prevalence at training 784 nodes}

785 (a) Scenario with training nodes having different prevalence: node 2 has only a 1:10 ratio.

786 Three prevalence scenarios are used in the test set. (b) Evaluation of scenario (a) showing 787 accuracy, sensitivity, specificity and F1 score. (c) Similar scenario as in (a) but prevalence 788 changed to 1:3 cases: controls in the training set. (d) Similar scenario as in (a) but prevalence 789 changed to 1:10 cases: controls in the training set. Box-whisker plot (mean, 1st and 3rd 790 quartile, whisker type Min/Max). Statistical differences between results derived by SL and 791 individual nodes including all permutations performed were calculated with Wilcoxon signed 792 rank test with continuity correction; asterisk and line: $p<0.05$. 
bioRxiv preprint doi: https://doi.org/10.1101/2020.06.25.171009; this version posted June 29, 2020. The copyright holder for this preprint (which was not certified by peer review) is the author/funder, who has granted bioRxiv a license to display the preprint in perpetuity. It is made available under aCC-BY-NC-ND 4.0 International license.

\section{Extended Data Figure 9}

a

$\mathrm{n}=2143,3$ training nodes, 1 test node

different prevalences

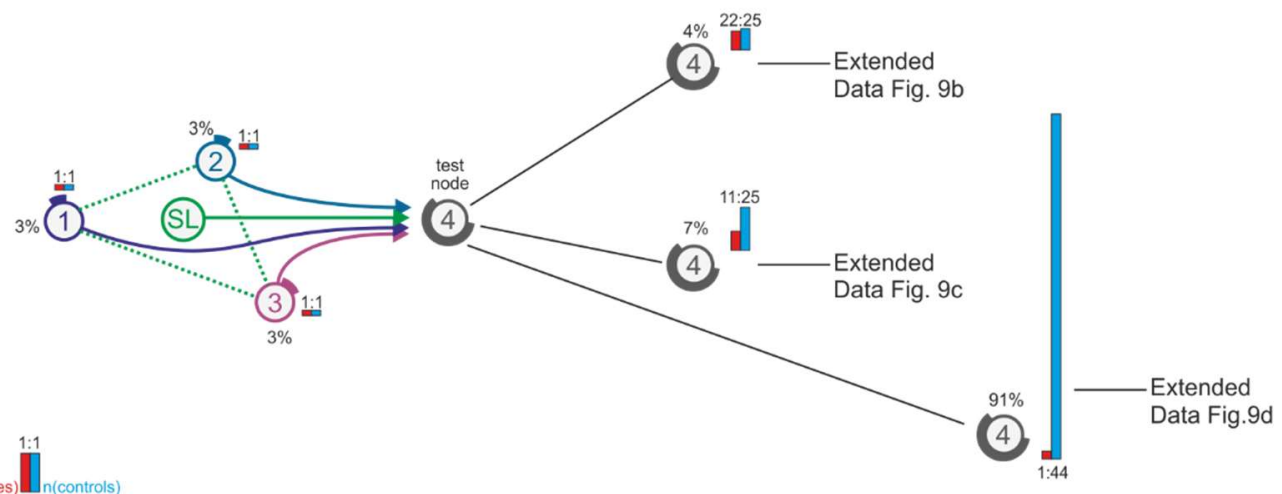

b

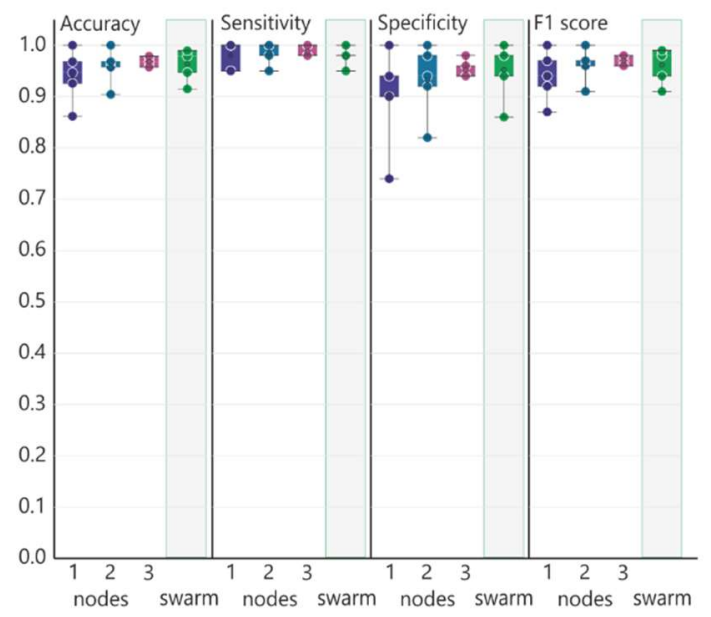

d

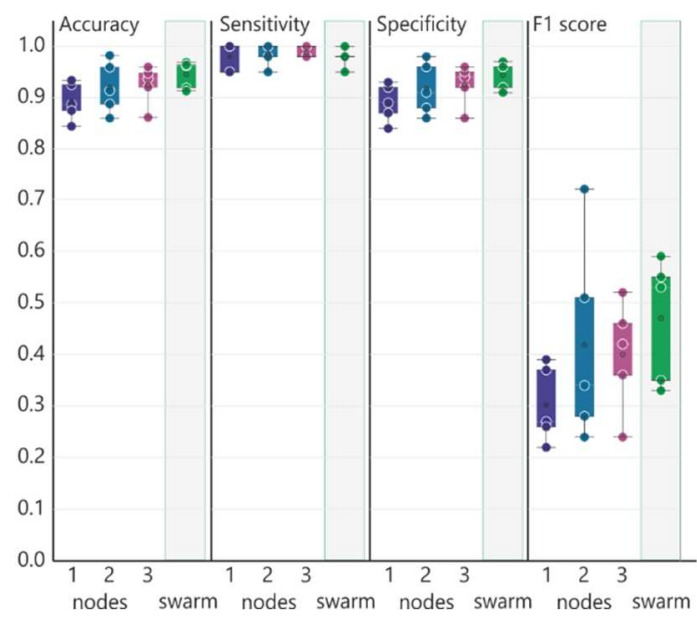

C

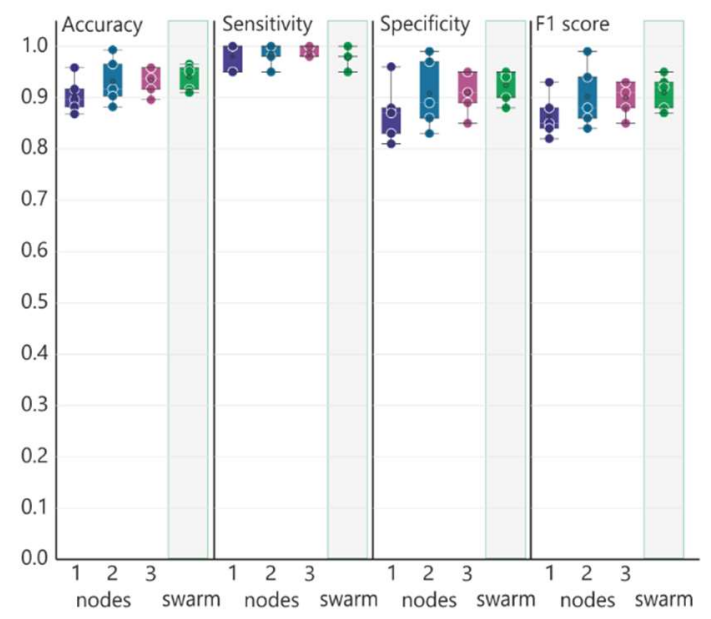




\section{Extended Data Figure 9. Baseline scenario for detecting COVID-19 patients}

796 (a) Scenario with even training set distribution among nodes 1-3. Three different testing sets

797 with different prevalence are simulated. (b) Evaluation of (a) for a 22:25 case: control ratio 798 showing accuracy, sensitivity, specificity and F1 score. (c) Evaluation results of scenario (a) 799 for a 11:25 ratio. (d) Evaluation results of scenario (a) for a 1:44 prevalence. Box-whisker plot 800 (mean, 1st and 3rd quartile, whisker type Min/Max). Statistical differences between results 801 derived by SL and individual nodes including all permutations performed were calculated with 802 Wilcoxon signed rank test with continuity correction; asterisk and line: $p<0.05$. 
bioRxiv reprint dol: https://doi.org/10.1101/2020.06.25.171009; this version posted June 29, 2020. The copyright holder for this preprint (which was not certified by peer review) is the author/funder, who has granted bioRxiv a license to display the preprint in perpetuity. It is

Extended Data Figure 10

a

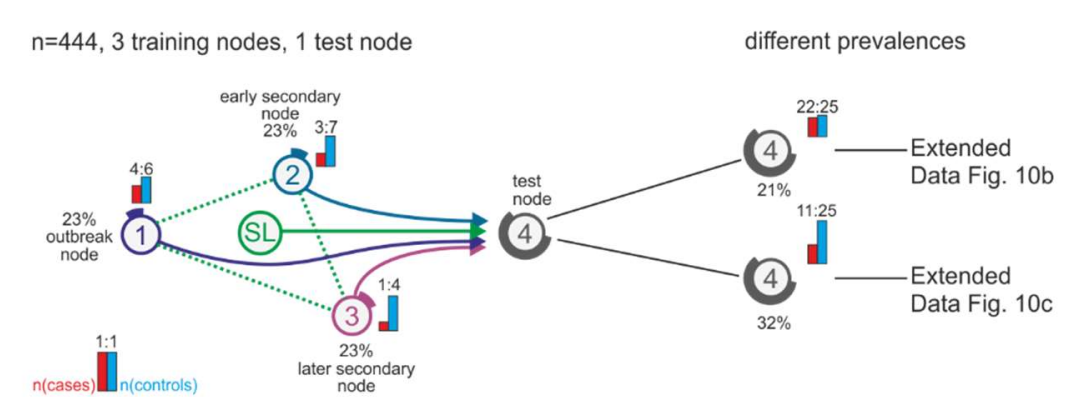

b

C
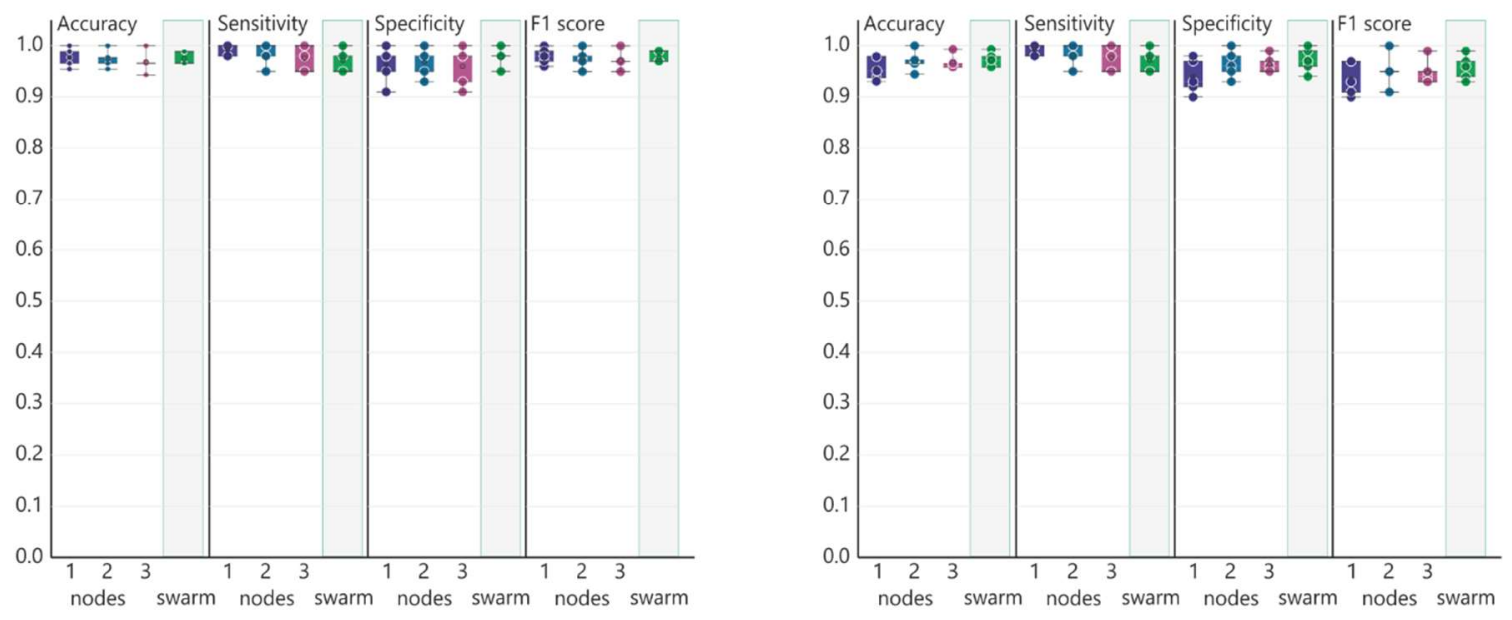

d

$\mathrm{n}=474,3$ training nodes, 1 test node

different prevalences

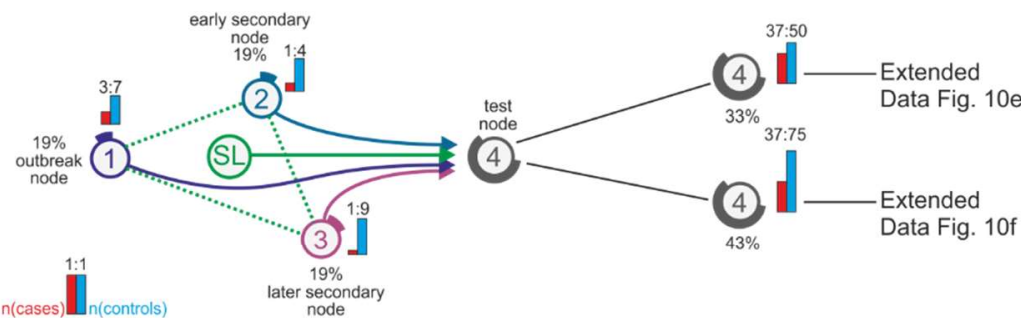

e

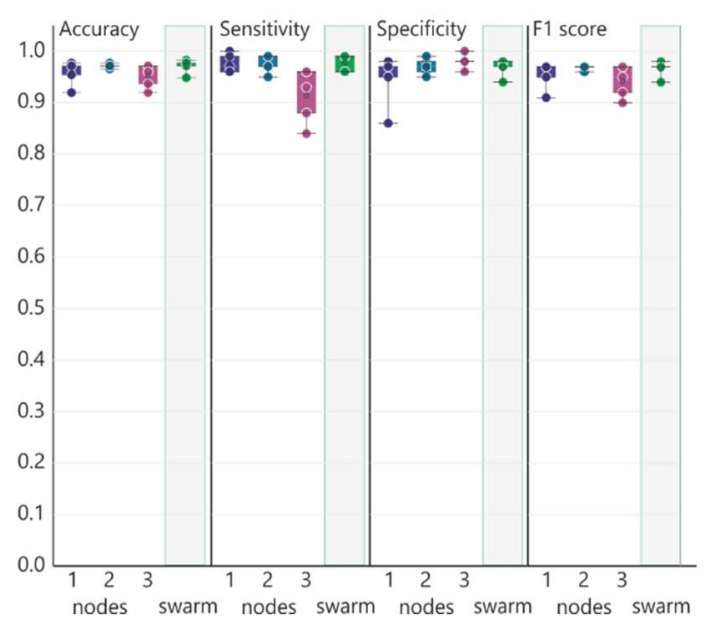

f

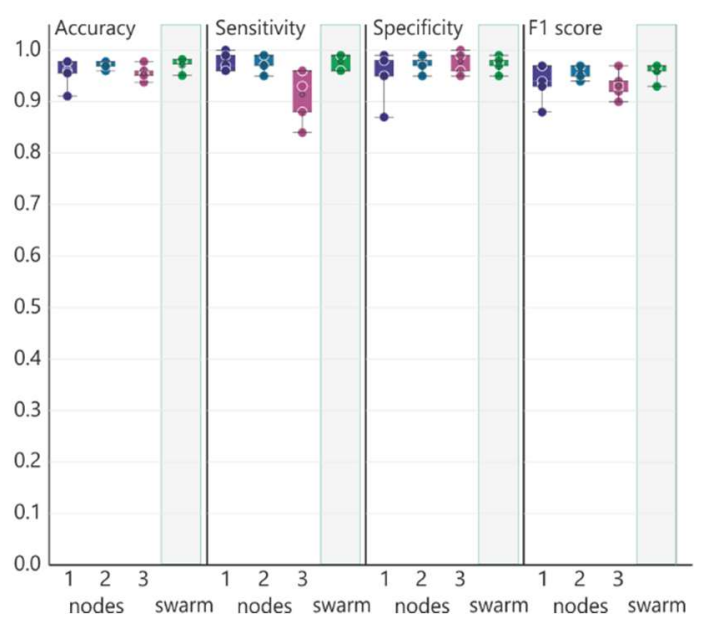

45 


\section{Extended Data Figure 10. Scenario with reduced prevalence at training nodes for 805 detecting COVID-19 patients}

806 (a) This scenario has the same sample size at each training node, but the prevalence

807 decreases from node 1 to node 3. There are two different test sets (b) and (c). (b) Evaluation 808 of scenario (a) with 22:25 ratio at the test node. (c) Results for the evaluation of scenario (a)

809 with reduced prevalence. (d) Scenario similar to (a) but the prevalence has a steeper decrease

810 between node 1 and 3. (e) Evaluation of scenario (d) with a ratio of 37:50 at the test node. (f)

811 Evaluation of (d) with a reduced prevalence compared to (e). Box-whisker plot (mean, 1st and

8123 3rd quartile, whisker type Min/Max). Statistical differences between results derived by SL and

813 individual nodes including all permutations performed were calculated with Wilcoxon signed

814 rank test with continuity correction; asterisk and line: $p<0.05$. 
bioRxiv preprint doi: https://doi.org/10.1101/2020.06.25.171009; this version posted June 29, 2020. The copyright holder for this preprint (which was not certified by peer review) is the author/funder, who has granted bioRxiv a license to display the preprint in perpetuity. It is made available under aCC-BY-NC-ND 4.0 International license.

\section{Extended Data Figure 11}

a

$\mathrm{n}=899,3$ training nodes, 1 test node different prevalences

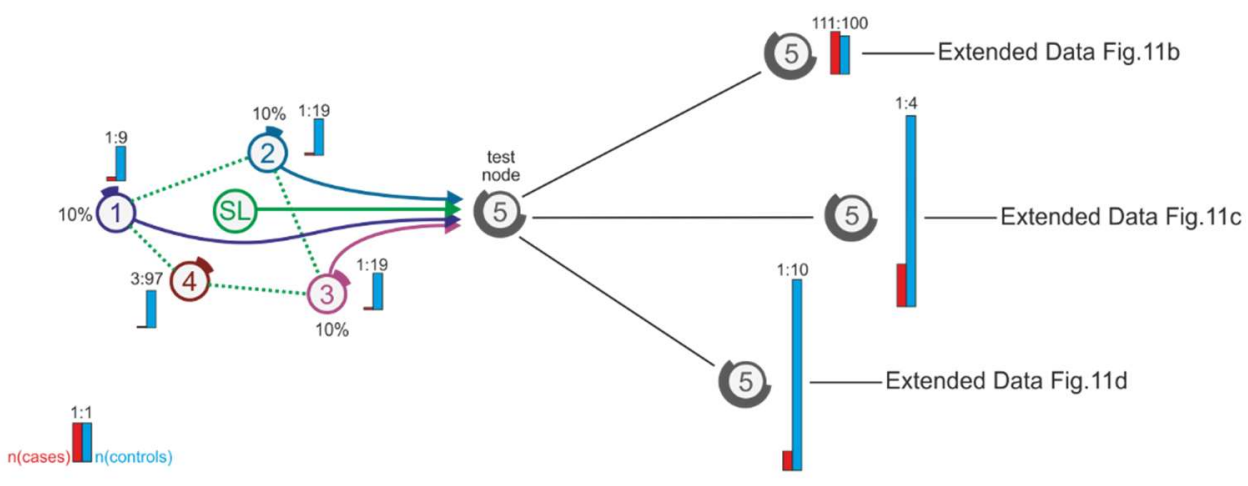

b

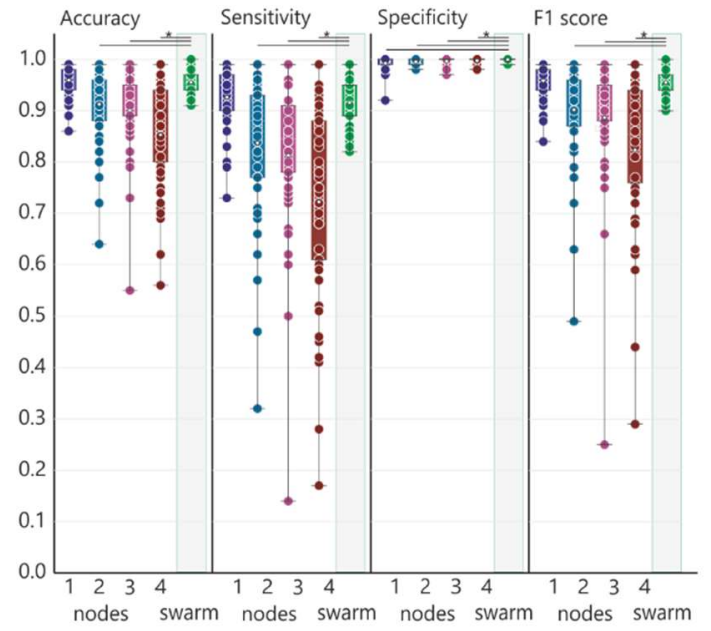

d

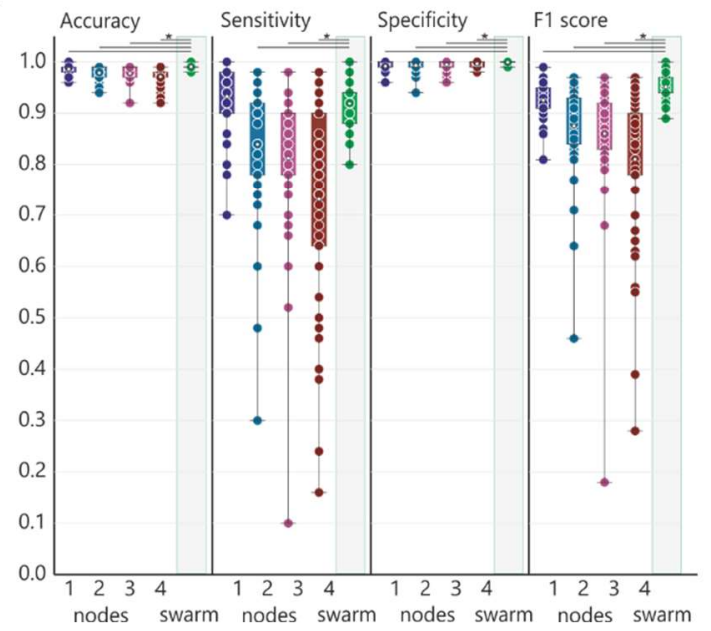

C

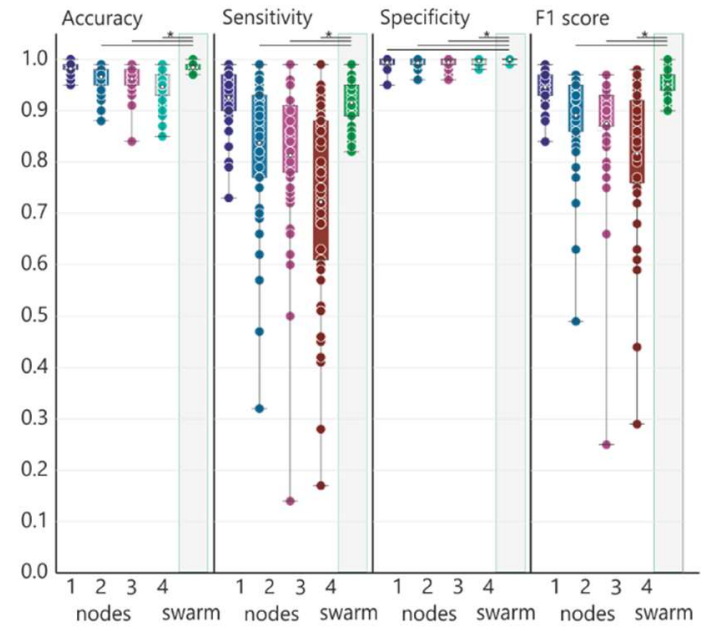




\section{Extended Data Figure 11. Scenario with reduced prevalence in training and test set at}

818 a 4-node setting (a) This scenario has even training set sizes among the nodes with the 819 prevalence ranging from $10 \%$ at node 1 to $3 \%$ at nodes 3 and 4 . There are three different test 820 sets (b), (c) and (d) with decreasing prevalence and increasing total sample size. (b) 821 Evaluation of scenario (a) with 111:100 ratio. (c) Evaluation of scenario (a) with 1:4 ratio and 822 increased sample number of the test set. (d) Results of scenario (a) with 1:10 prevalence and 823 increased sample number of the test set. Box-whisker plot (mean, 1st and 3rd quartile, whisker 824 type Min/Max). Statistical differences between results derived by SL and individual nodes 825 including all permutations performed were calculated with Wilcoxon signed rank test with 826 continuity correction; asterisk and line: $p<0.05$. 
828 (Material and Methods)

829

\section{Datasets}

832 We used a previously described dataset containing over 12,000 transcriptomes derived from 833 peripheral blood mononuclear cells (PBMC), deposited at the National Center for 834 Biotechnology Information Gene Expression Omnibus ${ }^{68}$ (GEO) under SuperSeries 835 GSE122517 or via the individual SubSeries GSE122505 (dataset 1), GSE122511 (dataset 2) 836 and GSE122515 (dataset 3). Briefly, this dataset was generated by inspection of all publicly 837 available datasets at GEO on September 20th, 2017. Inclusion criteria were cell type (PMBCs) 838 and species (Homo sapiens). Existing GEO SuperSeries were excluded to avoid duplicated 839 samples. According to data generation method, three datasets were established; dataset 1, 840 generated with Affymetrix HG-U133 A microarrays ( $n=2,500)$, dataset 2 with Affymetrix HG841 U133 2.0 microarrays $(n=8,348)$, and dataset 3 with high-throughput RNA sequencing (RNA842 seq $)(n=1,181)$. Data were curated as previously described ${ }^{47}$. All sample information is listed 843 in Supplementary Table 2.

846 To establish a dataset based on whole blood transcriptomes we generated new data from 847 healthy controls (Rhineland Study) and combined these with previously generated data that 848 had been deposited in Gene Expression Omnibus (GEO). We screened for transcriptome 849 datasets derived from human whole blood samples, which were collected using the PAXgene 850 Blood RNA System. In total, nine independent datasets were selected to be included in the present study (GSE101705 ( $n=44)$; GSE107104 $(n=33)$, GSE112087 $(n=120)$, GSE128078 ( $n=99)$, GSE66573 ( $n=14)$, GSE79362 ( $n=355)$, GSE84076 ( $n=36)$; GSE89403 ( $n=914))$. The newly generated 384 whole blood samples were sampled in context of the Rhineland Study led by the German Center for Neurodegenerative Diseases (DZNE), which is an extensive longitudinal study monitoring healthy individuals over 2 decades. Approval to undertake the Rhineland Study was obtained from the ethics committee of the University of Bonn, Medical Faculty. The study is carried out in accordance with the recommendations of the International Conference on Harmonization (ICH) Good Clinical Practice (GCP) standards (ICH-GCP). 
860

861

862

863

864

865

866

867

868

869

870

871

872

873

874

875

876

877

878

879

880

881

882

883

884

885

886

887

888

889

890

891

892

893

of Helsinki. Overnight fasting blood was collected from all participants, including a PAXgene ${ }^{\circledR}$ tube for RNA extraction and RNA-seq analysis. In total, Dataset B contained 1999 samples from patients with active tuberculosis $(n=775)$, latent tuberculosis $(n=277)$, fatigue $(n=55)$, autoimmune diseases $(n=68)$, HIV $(n=16)$ and controls $(n=808)$. Sample information is listed in Supplementary Table 2.

Whole blood derived transcriptome dataset for the prediction of COVID-19 (Dataset C)

To develop classifiers based on whole blood transcriptomes able to predict COVID-19 patients we collected an additional 134 PAXgene $₫$ tubes for RNA extraction and RNA-seq analysis from COVID-19 patients, of which 93 whole blood samples at the Intensive Care Unit of the Radboud University Medical Centre in Nijmegen, the Netherlands, and 41 samples were either collected at the Sotiria Athens General Hospital or the ATTIKON University General Hospital in Athens, Greece. For all COVID-19 patients, the study was carried out in accordance with the applicable rules concerning the review of research ethics committees and informed consent. All patients or legal representatives were informed about the study details and could decline to participate. COVID-19 was diagnosed by a positive SARS-CoV-2 RT-PCR test in nasopharyngeal or throat swabs and/or by typical chest CT-scan finding. Blood for RNA-seq analysis was sampled on day 0 to 11 after admission. In the cohort in Athens, blood samples from ten healthy donors who were tested negative on SARS-CoV-2 were included as controls. The newly generated samples from the COVID-19 patients and the controls from Athens were combined with dataset $B$ (see above) to establish Dataset $C$. As a result, in addition to the 1999 samples derived from Dataset B, Dataset C included further 10 healthy controls and 134 dutch COVID-19 samples, which makes a total of 2,143 samples. Sample information is listed in Supplementary Tables 2 and 6.

\section{Pre-processing}

\section{PBMC transcriptome dataset (Dataset A)}

We used a previously published dataset compiled for predicting AML in blood transcriptomes derived from peripheral blood mononuclear cells (PBMC) ${ }^{47}$. Briefly, all raw data files were downloaded from GEO and the RNA-seq data was preprocessed using the kallisto aligner against the human reference genome gencode v27 (GRCh38.p10). For normalization, we considered all platforms independently, meaning that normalization was performed separately for the samples in Dataset A1, A2 and A3, respectively. Microarray data (Datasets A1 and A2) 
894 was normalized using the robust multichip average (RMA) expression measures ${ }^{69}$, as 895 implemented in the R package affy ${ }^{70}$. RNA-seq data (Dataset A3) was normalized with the R 896 package DESeq2 using standard parameters ${ }^{71}$. In order to keep the datasets comparable, 897 data was filtered for genes annotated in all three datasets, which resulted in 12,708 genes. No 898 filtering of low-expressed genes was performed. All scripts used in this study for pre899 processing are provided as a docker container on Docker Hub (docker hub, 900 https://hub.docker.com/r/schultzelab /aml_classifier).

901

902 Whole blood derived transcriptome datasets (Datasets B and C)

903 Since alignment of whole blood transcriptome data can be performed in numerous different 904 ways, we re-aligned all downloaded and collected datasets which were 4.7 Terabyte in size 905 and comprised a total of 7.8 Terabases, to the human reference genome gencode v33 906 (GRCh38.p13) and quantified transcript counts using STAR, an ultrafast universal RNA-seq 907 aligner (version 2.7.3a) ${ }^{72}$. For all samples in Datasets $B$ and $C$, raw counts were imported 908 using DESeqDataSetFromMatrix function and size factors for normalization were calculated 909 using the DESeq function using standard parameters ${ }^{71}$. This was done separately for Dataset

910 B and Dataset C. Since some of the samples were prepared with poly-A selection to enrich 911 for protein-coding mRNAs, we filtered the complete dataset for protein-coding genes in order 912 to ensure greater comparability across library preparation protocols. Furthermore, we 913 excluded all ribosomal protein-coding genes, as well as mitochondrial genes and genes coding 914 for hemoglobins, which resulted in 18,135 transcripts as the feature space in Dataset B and 91519,358 transcripts in Dataset C. Furthermore, transcripts with an overall expression < 10 were 916 excluded from further analysis. Other than that, no filtering of transcripts was performed. Prior 917 to use in machine learning we performed a rank transformation to normality on both datasets $918 B$ B and $C^{73}$. Briefly, transcript expression values were transformed from RNAseq counts to their 919 respective ranks. This was done transcript-wise, meaning all transcript expression values per 920 sample were given a rank based on ordering them from lowest to highest value. The rankings 921 were then turned into quantiles and transformed via the inverse cumulative distribution 922 function of the Normal distribution. This leads to all transcripts following the exact same 923 distribution (that is, a standard Normal with a mean of 0 and a standard deviation of 1 ) across 924 all samples

925 Methods details 
927 We previously demonstrated that ML on PBMC transcriptomes can be utilized to predict $928 \mathrm{AML}^{47}$. Based on this experience, we generated sample sets within three independent 929 transcriptome datasets (dataset A1-A3, see above) to assess different scenarios in a three930 node setting for training with a fourth node only used for testing. As indicated in Fig. 2, six 931 scenarios with varying numbers of samples per node and varying ratios between cases and 932 controls at each node where defined. For predicting AML, all samples derived from AML 933 patients were classified as cases, while all other samples were labeled controls. When 934 predicting ALL, all samples derived from ALL patients were classified as cases and all others 935 as controls. For each scenario (Fig. 2) and each dataset we permuted the sample distribution 936100 times, resulting in a total of 5,594 individual predictions. The different scenarios were 937 chosen to address the influence of sample numbers per node, the case control ratio, study 938 design-related batch effects, and transcriptome technologies used on classifier performance 939 at the nodes, but more importantly on swarm learning performance. Sample distributions for 940 all permutations within all scenarios are listed in Supplementary Table 1.

941

\section{Scenarios for detecting patients with acute TB}

943 In line with the experience we gained from the prediction of $A M L$, we used dataset $B$ to 944 generate scenarios for the prediction of tuberculosis in various settings, again using different 945 scenarios in a three-node setting for training with a fourth node only used for testing. In one 946 scenario, all patients with tuberculosis ( $\mathrm{Tb}$ ) including patients with latent and acute Tb were 947 treated as cases, while all others were defined as controls (Extended Data Fig. 6b). In all 948 other scenarios, cases were restricted to acute Tb patients' samples, while patients with latent $949 \mathrm{~Tb}$ were defined as controls together with all other non-Tb samples. Here, the question to be 950 answered is, whether the classifiers can identify patients with acute $\mathrm{Tb}$ and can distinguish 951 them from latent $\mathrm{Tb}$ and other conditions.

952 In one scenario (Fig. 3c-d), we added three additional training nodes to test dependency of 953 classifier performance by the number of nodes. As indicated in Fig. 3, three scenarios with 954 varying numbers of samples per node and varying ratios between cases and controls at each 955 node where defined. For scenarios described within Fig. 3e,g and Fig. 3i,k, we tested two 956 prevalence scenarios in the test set. For each scenario (Fig. 3) we permuted the sample 957 distribution 5-10 times, resulting in a total of 325 individual predictions. To mimic an outbreak 958 scenario, we reduced cases also at the training nodes to determine the effects on Swarm 959 Learning performance. Sample distributions for all permutations within all scenarios are listed 960 in Supplementary Table 1. 


\section{Simulation of an outbreak scenario to detect COVID-19 patients}

963 Based on the promising results obtained with tuberculosis, we next intended to simulate 964 classifier building and testing for the prediction of COVID-19 in a SL setting. We used dataset

965 B and added 144 additional samples, of which 139 samples were derived from COVID-19 966 patients (see above). We applied a three-node setting for training with a fourth node only used 967 for testing.

968 In one scenario (Extended Data Fig. 8), we kept cases $(n=30)$ and controls $(n=30)$ evenly 969 distributed among the three training nodes and tested three different prevalence scenarios at 970 the test node $(22: 25 ; 11: 25 ; 1: 44)$. In a second scenario (Extended Data Fig. 9a-c) we 971 changed the ratio of cases and controls at each node (node 1: 40:60, node 2: 30:70, node 3 : $97220: 80)$ and tested two prevalence scenarios at the test node $(22: 25 ; 11: 25)$. In a third scenario

973 (Extended Data Fig. 9a-c) we further reduced the number of cases at the training nodes 974 further (node 1: 30:70, node 2: 20:80, node 3: 10:90) and tested two prevalence scenarios at 975 the test node $(37: 50 ; 37: 75)$.

976 Lastly, we tested an outbreak scenario (Fig. 4) with very few cases at the outbreak node 1 977 (20:80), an early secondary node (10:90) and a later secondary node (5:95) and three 978 prevalence scenarios at the test node $(1: 1,1: 2,1: 10)$, resulting in a total of 220 individual 979 predictions Sample distributions for all permutations within all scenarios are listed in 980 Supplementary Table 1.

981

982

983

984

985

986

987

988

989

990

991

992

993

\section{Application layer}

The application layer (see also Fig. 1g) consists of disease models for which definitions are given, which samples are cases and which samples are controls. For example, if the classifier is supposed to detect all patients with tuberculosis $(\mathrm{Tb})$, the model includes patients with latent and acute tuberculosis as cases and all other samples as controls. However, if only patients with acute tuberculosis are intended to be detected as cases, the model is changed in that cases are now only patient samples derived from patients with acute $\mathrm{Tb}$, while samples from patients with latent Tb are now treated as controls, similar to all other non-Tb samples. The cases and controls used for each scenario are given in the result section in more detail. For each mode, classifiers are generated by applying neural networks (for description see below)

\section{Computation and analysis}

\section{Neural network algorithm}


994 We leveraged a deep neural network with a sequential architecture as implemented in the

995 keras library (Keras, https://keras.io/, 2015). Briefly, the neural network consists of one input

996 layer, eight hidden layers and one output layer. The input layer is densely connected and

997 consists of 256 nodes, a rectified linear unit activation function and a dropout rate of $40 \%$.

998 From the first to the eighth hidden layer, nodes are reduced from 1024 to 64 nodes, and all

999 layers contain a rectified linear unit activation function, a kernel regularization with an L2

1000 regularization factor of 0.005 and a dropout rate of $30 \%$. The output layer is densely connected

1001 and consists of 1 node and a sigmoid activation function. The model is configured for training

1002 with Adam optimization and to compute the binary cross-entropy loss between true labels and

1003 predicted labels.

1004 The model has been translated from $\mathrm{R}$ to Python in order to make it compatible with the swarm

1005 learning library. This model is used for training both the individual nodes as well as swarm

1006 learning. The model is trained over 100 epochs, with varying batch sizes. The batch size of 8 ,

1007 16, 32, 64 and 128 are used depending on the number of training samples.

1008

1009 Preparation and adaptation of neural network code to be used in a swarm learning 1010 environment

1011 A swarm callback is introduced to integrate the model with the Swarm Learning library.

1012 Minimum number of nodes for synchronization, synchronization interval, validation dataset 1013 and batch size are passed as parameters to swarm callback. The swarm call back API is

1014 swCallback $=$ SwarmCallback (sync_interval $=<$ number of training batches between syncs $>$, min_peers $=\langle$ minimum peers $>$, val data $=\langle$ validation dataset $>$, val_batch_size $=<$ validation batch size $>$, node_weightage $=<$ relative weightage of node's model weights $>$ )

1018

1019 sync_interval specifies the synchronization interval,

1020 min_peers specifies the minimum number of nodes for model synchronization,

1021 val_data specifies the validation data set,

1022 val_batch_size specifies the validation batch size,

1023 model_name specifies the name of the model,

1024 node_weightage specifies the relative weightage to be given to model weights of this node

1025

1026 Parameter tuning

1027 For some of the scenarios we tuned model hyperparameters. For some scenarios we also 1028 tuned Swarm Learning parameters to get better performance, for example higher sensitivity. 
1029 For AML Fig. 2e, Extended Data Fig. 2 and Fig. 2f, dropout rate is reduced to $10 \%$ to get 1030 better performance. For AML Fig. 2b, Extended Data Fig. 1, dropout rate is reduced to 10\% and increased the Epochs to 300 to get better performance. We also used the adaptive_rv parameter in the Swarm Learning API to adjust the merge frequency dynamically based on model convergence to improve the training time. For TB and COVID-19 tests dropout rate is reduced to $10 \%$ for all scenarios. For the TB scenarios in Extended Data Fig. 7a,b, the node_weightage parameter of Swarm Learning callback API is used to give more weightage to the nodes that have higher case samples.

Infrastructure layer

\section{Description of the hardware architecture applied for simulations}

1040 For all simulations provided in this project we used 2 HPE Apollo 6500 Gen 10 server, each with 4 Intel(R) Xeon(R) CPU E5-2698 v4 @ 2.20GHz, a 3.2 TB hard disk drive, 256 GB RAM, 8 Tesla P100 GPUs, 1GB network interface card for LAN access and infiniBand FDR for high speed interconnect and networked storage access. The Swarm Network is created with 3 nodes, each node is a docker container with $1 \mathrm{GPU}$. Multiple experiments were run in parallel using the above described configuration.

1046 Overall, we performed 6,139 analyses including six scenarios for all three AML datasets, nine scenarios for Tb and 10 scenarios for COVID-19. We performed 5 to 100 permutations per scenario, each permutation took approximately 30 minutes, which resulted in a total of 3069,5 compute hours.

1050

Swarm Learning builds on top of two proven technologies — distributed ML and blockchain. Distributed ML is leveraged to train a common model across multiple nodes with a subset of the data located at each node - commonly known as the data parallel paradigm in $\mathrm{ML}-$ though without a central parameter server. Blockchain lends the decentralized control, scalability, and fault-tolerance aspects to the Swarm Network system to enable the framework to work beyond the confines of a single enterprise. a set of nodes - each node possessing some training data locally - to train a common ML 
1061

1062

1063

1064

1065

1066

1067

1068

1069

1070

1071

1072

1073

1074

1075

1076

1077

1078

1079

1080

1081

1082

1083

1084

1085

1086

1087

1088

1089

1090

1091

model collaboratively without sharing the training data itself. This can be achieved by individual nodes sharing parameters (weights) derived from training the model on the local data. This allows nodes to maintain the privacy of their raw data. Importantly, in contrast to many existing federated learning models, a central parameter server is omitted in Swarm Learning.

The nodes that participate in Swarm Learning, register themselves with the Swarm Network implicitly using the callback API. Here, the Swarm Network interacts with other peers using blockchain for sharing parameters and for controlling the training process. On each node, a simple Swarm callback API has to be used to enable the ML model with Swarm Learning capacities (see also code presented below). The Swarm container has to be configured to interact with the Swarm Network (network i/p and port configuration). All other complexities of setting up network, registration, parameter sharing, and parameter merging are taken care of by the Swarm callback API and the Swarm Network infrastructure.

Parameters shared from all the nodes are merged to obtain a global model. Moreover, the merge process is not done by a static central coordinator or parameter server, but rather a temporary leader chosen dynamically among the nodes is used to perform the merge, thereby making the Swarm network decentralized. This provides a far greater fault-tolerance than traditional centralized-parameter-server-based frameworks. All the nodes can perform the role of training and merging, thereby maximising the usage of local compute. The Swarm Network implicitly controls this.

The HPE Swarm Learning library contains 2 containers, the Swarm Network container and the Swarm ML container.

The Swarm Network container includes 1) software to setup and initialize the Swarm Network, 2) management commands to control the Swarm Network, and 3) start/stop Swarm Learning tasks. This container also encapsulates the blockchain software.

The Swarm ML container includes software to support 1) decentralized training, 2) integration with ML frameworks, and 3) it exposes APIs for ML models to interact with Swarm Learning.

For any ML model to be applied to Swarm Learning, it needs to be modified using the Swarm callback API. The callback API provides options to control the Swarm Learning processes. To convert a ML program into a Swarm ML program the following steps have to be performed:

1. Import the SwarmCallback class from the swarm library

from swarm 'import SwarmCallback' 
2. Instantiate an object of the SwarmCallback class: swarm_callback $=$ SwarmCallback $($ min_peers $=\langle$ peer count $\rangle$, sync_interval $=<$ interval $>$, use_adaptive_sync $=\langle$ bool $>$, val_batch_size $=\langle$ batch size $>$, val_data $=<$ either a (x_val, y_val) tuple or a generator $>$ node_weightage $=$ <relative weightage of node's model weights $>$ ).

In this context, 'min_peers' specifies the minimum number of network peers required to synchronize the insights, 'sync_interval' specifies the number of batches after which a synchronization is performed, 'use_adaptive_sync' specifies whether the adaptive sync interval feature should be used for tuning the sync interval. This feature is turned off by default; 'val_batch_size' specifies the size of each validation batch; 'val_data' specifies the validation dataset. It can be either a (x_val, y_val) tuple or a generator;

3. Pass the object to the list of callbacks in Keras training code: model.fit(..., callbacks $=$ [swarm_callback]). SwarmCallback can be included along with other callbacks also:

es_callback = EarlyStopping $(\ldots)$;

model.fit(..., callbacks $=$ [es_callback, swarm_callback $])$

\section{The Swarm Learning architecture principles}

1115 The Swarm Learning framework has two major components, 1) the Swarm ML component 1116 runs a user-defined Machine Learning algorithm, and 2) the Swarm Network component forms 1117 the Swarm Network based on a blockchain network.

1118 The Swarm ML component is implemented as an API available for multiple popular 1119 frameworks such as TensorFlow, Keras, Pytorch. This API provides an interface that is similar 1120 to the training APIs in the native frameworks familiar to data scientists. Calling this API 1121 automatically inserts the required hooks for Swarm Learning so that nodes seamlessly 1122 exchange parameters and subsequently continue the training after setting the local models to 1123 the globally merged parameters. With a few simple code changes, the entire network learns 1124 as one cohort, with all the complexities of control and data flow taking place in an automated fashion. 
1126 Within the Swarm Network component each Swarm ML component interacts with each other 1127 using the Swarm Network component's blockchain platform to maintain global state 1128 information about the model that is being trained and to track the training progress. The Swarm 1129 Network components use this state and progress information to coordinate the working of the 1130 Swarm learning. The Swarm Network is responsible for keeping the decentralized Swarm 1131 network in a globally consistent state. The Swarm Network ensures that all operations and the 1132 corresponding state transitions are performed in a synchronous manner. Both, state and 1133 supported operations of the system are encapsulated in a blockchain smart contract. The 1134 Swarm Network contains the logic to elect the leader of the Swarm for every synchronization, 1135 implement fault-tolerance, and self-healing mechanisms, along with signaling among nodes 1136 for commencement and completion of various phases.

1137 The Swarm Learning framework is designed to run on both commodity and high-end 1138 machines, supporting a heterogeneous set of infrastructure in the network. It can be deployed 1139 within and across data centers.

1140 In contrast to federated learning with star topology and a centralized coordinator, Swarm 1141 Learning can support multiple topologies including fully connected, mesh, star, tree and hybrid 1142 topologies. This flexibility provides multiple options to cater into different use cases.

The Swarm Learning process

1145 Swarm Learning provides a callback API to enable swift integration with multiple frameworks. 1146 This API is incorporated into the existing ML code to quickly transform a stand-alone ML node 1147 into a Swarm Learning participant in a non-intrusive way. It offers a set of commands (APIs) 1148 to manage the Swarm Network and control the training.

1149 The Swarm learning process is as follows:

1150 The Swarm Learning process begins with enrollment of nodes with Swarm Network, which is 1151 done implicitly by Swarm callback function when the callback is constructed. During this 1152 process, the relevant attributes of the node are stored in the blockchain ledger. This is a one1153 time process.

1154 Nodes will train the local copy of the model iteratively using private data over multiple epochs. 1155 During each epoch, the node trains its local model using one or more data batches for a fixed 1156 number of iterations. It regularly shares its learnings with the other Swarm nodes and 1157 incorporates their insights. Users can control the periodicity of this sharing by defining a 
1158 Synchronization Interval in Swarm callback API. This interval specifies the number of training 1159 batches after which the nodes will share their learnings.

1160 At the end of every synchronization interval, when it is time to share the learnings from the 1161 individual models, one of the Swarm nodes is elected as a "leader" using the leader election 1162 logic. This leader node collects the model parameters from each peer node and merges them. 1163 The framework supports multiple merge algorithms such as mean, weighted mean, median, 1164 and so on. Each node then uses these merged parameters to calculate various validation 1165 metrics. These results are compared against the stopping criterion and if it is found to be met, 1166 the Swarm Learning process is halted. Else the nodes use the merged parameters to start the 1167 next training batch.

1168 Swarm Learning library uses blockchain smart contracts to define the leader election logic and 1169 the merge algorithm. The blockchain smart contracts prevents attacks from semi-honest or 1170 dishonest participants.

\section{Quantification and Statistical Analysis}

1173 We evaluated binary classification model performance with sensitivity, specificity, accuracy 1174 and f1-score metrics. Sensitivity, specificity, accuracy and f1-score were determined for every 1175 test run. The 95\% confidence intervals of all performance metrices were estimated using the 1176 boostrapping approach ${ }^{74}$. For $A M L$ and ALL, 100 permutations per scenario were run for each 1177 scenario. For TB the performance metrics were collected by running 10 permutations for 1178 scenarios 1 to 4 and 5 permutations for scenarios 5 to 10. For COVID-19 the performance 1179 metrics were collected by running 20 permutations for each scenario. All metrics are listed in 1180 Supplementary Tables 3 and 4.

1181 Differences in performance metrics were tested using the Wilcoxon signed rank test with 1182 continuity correction (Individual Comparisons by Ranking Methods, Frank Wilcoxon, 1183 https://sci2s.ugr.es/keel/pdf/algorithm/articulo/wilcoxon1945.pdf). All test results are provided in Supplementary Table 5.

1185 To run the experiments, we used Python version 3.6.9 with Keras version 2.3.1 and 1186 Tensorflow version 2.2.0-rc2. We used scikit-learn library version $0.23 .1^{75}$ to calculate values 1187 for the metrics. Summary statistics and hypothesis tests were calculated using $\mathrm{R}$ version 3.5.2 1188 (R: A language and environment for statistical computing, http://www.R-project.org/., 2015). 1189 Calculation of each metric was done as follows: 


$$
\text { Sensitivity }=\frac{T P}{T P+F N}
$$

$$
\text { Specificity }=\frac{T N}{T N+F P}
$$

$$
\text { Accuracy }=\frac{T P+T N}{T P+F P+T N+F N}
$$

$$
\text { Balanced Accuracy }=\frac{\text { Sensitivity }+ \text { Specificity }}{2}
$$

$$
F 1-\text { score }=\frac{2 T P}{F P+F N+2 T P}
$$

where TP=True Positive, FP=False Positive, $T N=$ True Negative, FN=False Negative

\section{Data visualization}

The classification report and confusion matrix was generated with scikit-learn APIs for each permutation. Measurements of sensitivity, specificity and accuracy of each permutation run was read into a table in Excel using Power Query and used for visualization for the different scenarios in Power BI [Version: 2.81.5831.821 64-bit (Mai 2020)] with Box and Whisker chart by MAQ Software (https://appsource.microsoft.com/en-us/product/power-bi-visuals/ WA104381351).

\section{Data and software availability:}

1206 Processed data can be accessed via the SuperSeries GSE122517 or via the individual 1207 SubSeries GSE122505 (dataset A1), GSE122511 (dataset A2) and GSE122515 (dataset A3). 1208 Dataset $B$ consists of the following series which can be accessed at GEO: GSE101705, 1209 GSE107104, GSE112087, GSE128078, GSE66573, GSE79362, GSE84076, and GSE89403. 1210 Furthermore, it contains the Rhineland study. This dataset is not publicly available because of 1211 data protection regulations. Access to data can be provided to scientists in accordance with 1212 the Rhineland Study's Data Use and Access Policy. Requests for further information or to 1213 access the Rhineland Study's dataset should be directed to RS-DUAC@dzne.de. Dataset C 1214 contains dataset B and additional samples for COVID-19. These datasets are made available 1215 at the European Genome-Phenome Archive (EGA) under accession number 1216 EGAS00001004502, which is hosted by the EBI and the CRG. 
1217 The code for preprocessing and for predictions can be found at GitHub 1218 (https://github.com/schultzelab/swarm_learning).

\section{Supplementary Tables}

1222 Supplementary Table 1:

Overview over all sample numbers and scenarios

1223 Supplementary Table 2:

Dataset annotations of Dataset A, B and C

1224 Supplementary Table 3:

Prediction results for all scenarios and permutations

1225 Supplementary Table 4:

Summary statistics on all prediction scenarios

1226 Supplementary Table 5: Statistical tests comparing single node vs. swarm predictions

1227 Supplementary Table 6: Covid 19 Patient characteristics 


\section{References}

1. Haendel, M. A., Chute, C. G. \& Robinson, P. N. Classification, ontology, and precision medicine. N. Engl. J. Med. 379, 1452-1462 (2018).

2. Aronson, S. J. \& Rehm, H. L. Building the foundation for genomics in precision medicine. Nature 526, 336-342 (2015).

3. Courtiol, P. et al. Deep learning-based classification of mesothelioma improves prediction of patient outcome. Nat. Med. 25, 1519-1525 (2019).

4. Liang, $\mathrm{H}$. et al. Evaluation and accurate diagnoses of pediatric diseases using artificial intelligence. Nat. Med. 25, 433-438 (2019).

5. Wiens, J. et al. Do no harm: a roadmap for responsible machine learning for health care. Nat. Med. 25, (2019).

6. He, J. et al. The practical implementation of artificial intelligence technologies in medicine. Nat. Med. 25, 30-36 (2019).

7. Babic, B., Gerke, S., Evgeniou, T. \& Glenn Cohen, I. Algorithms on regulatory lockdown in medicine. Science 366, 1202-1204 (2019).

8. Price, W. N. \& Cohen, I. G. Privacy in the age of medical big data. Nat. Med. 25, 37-43 (2019).

9. Zhang, X. et al. Viral and host factors related to the clinical outcome of COVID-19. Nature (2020) doi:10.1038/s41586-020-2355-0.

10. Berlin, D. A., Gulick, R. M. \& Martinez, F. J. Severe Covid-19. N. Engl. J. Med. (2020) doi:10.1056/nejmcp2009575.

11. Gandhi, R. T., Lynch, J. B. \& del Rio, C. Mild or Moderate Covid-19. N. Engl. J. Med. (2020) doi:10.1056/nejmcp2009249.

12. Cho, A. Al systems aim to sniff out coronavirus outbreaks. Science $368,810-811$ (2020).

13. Peiffer-Smadja, N. et al. Machine Learning for COVID-19 needs global collaboration and data-sharing. Nat. Mach. Intell. 1-2 (2020) doi:10.1038/s42256-020-0181-6.

14. Luengo-Oroz, M. et al. Artificial intelligence cooperation to support the global response to COVID-19. Nat. Mach. Intell. 1-3 (2020) doi:10.1038/s42256-020-0184-3.

15. Hu, Y. et al. The challenges of deploying artificial intelligence models in a rapidly evolving pandemic. Nat. Mach. Intell. 2, 298-300 (2020).

16. Senior, A. W. et al. Improved protein structure prediction using potentials from deep learning. Nature 577, 706-710 (2020).

17. Ge, Y. et al. A data-driven drug repositioning framework discovered a potential therapeutic agent targeting COVID-19. bioRxiv (2020) doi:10.1101/2020.03.11.986836.

18. Ting, D. S. W., Carin, L., Dzau, V. \& Wong, T. Y. Digital technology and COVID-19. Nat. Med. 26, 459-461 (2020).

19. Cohen, I. G., Gostin, L. O. \& Weitzner, D. J. Digital Smartphone Tracking for COVID19: Public Health and Civil Liberties in Tension. JAMA (2020) doi:10.1001/jama.2020.8570.

20. Jia, J. S. et al. Population flow drives spatio-temporal distribution of COVID-19 in China. Nature (2020) doi:10.1038/s41586-020-2284-y.

21. Mei, X. et al. Artificial intelligence-enabled rapid diagnosis of patients with COVID-19. Nat. Med. (2020) doi:10.1038/s41591-020-0931-3. 
22. Zhang, K. et al. Clinically Applicable Al System for Accurate Diagnosis, Quantitative Measurements, and Prognosis of COVID-19 Pneumonia Using Computed Tomography. Cell 181, (2020).

23. Amisha, Malik, P., Pathania, M. \& Rathaur, V. Overview of artificial intelligence in medicine. J. Fam. Med. Prim. Care 8, 2328 (2019).

24. Hosny, A., Parmar, C., Quackenbush, J., Schwartz, L. H. \& Aerts, H. J. W. L. Artificial intelligence in radiology. Nature Reviews Cancer. 18, 500-510 (2018).

25. Lecun, Y., Bengio, Y. \& Hinton, G. Deep learning. Nature. 521, 436-444 (2015).

26. Kaissis, G. A., Makowski, M. R., Rückert, D. \& Braren, R. F. Secure, privacy-preserving and federated machine learning in medical imaging. Nat. Mach. Intell. (2020) doi:10.1038/s42256-020-0186-1.

27. Rajkomar, A., Dean, J. \& Kohane, I. Machine learning in medicine. New England Journal of Medicine vol. 380 1347-1358 (2019).

28. Obermeyer, Z. \& Emanuel, E. J. Predicting the future-big data, machine learning, and clinical medicine. New England Journal of Medicine. 375, 1216-1219 (2016).

29. Savage, N. Machine learning: Calculating disease. Nature. 550, S115-S117 (2017).

30. Ping, P., Hermjakob, H., Polson, J. S., Benos, P. V. \& Wang, W. Biomedical informatics on the cloud: A treasure hunt for advancing cardiovascular medicine. Circ. Res. 122, 1290-1301 (2018).

31. Chouvarda, I. et al. WELCOME - Innovative integrated care platform using wearable sensing and smart cloud computing for COPD patients with Comorbidities. in 2014 36th Annual International Conference of the IEEE Engineering in Medicine and Biology Society, EMBC 2014 vol. 2014 3180-3183 (Institute of Electrical and Electronics Engineers Inc., 2014).

32. Grossman, R. L. \& White, K. P. A vision for a biomedical cloud. in Journal of Internal Medicine. 271, 122-130 (J Intern Med, 2012).

33. Char, D. S., Shah, N. H. \& Magnus, D. Implementing machine learning in health care ' addressing ethical challenges. New England Journal of Medicine. 378, 981-983 (2018).

34. Gibney, E. The battle for ethical Al at the world's biggest machine-learning conference. Nature. 577, 609 (2020).

35. Finlayson, S. G. et al. Adversarial attacks on medical machine learning. Science. 363, 1287-1289 (2019).

36. Ienca, M. \& Vayena, E. On the responsible use of digital data to tackle the COVID-19 pandemic. Nat. Med. 26, 463-464 (2020).

37. Konečný, J., McMahan, H. B., Ramage, D. \& Richtárik, P. Federated Optimization: Distributed Machine Learning for On-Device Intelligence. (2016).

38. Konečný, J. et al. Federated Learning: Strategies for Improving Communication Efficiency. (2016).

39. McMahan, H. B., Moore, E., Ramage, D., Hampson, S. \& Arcas, B. A. y. Communication-Efficient Learning of Deep Networks from Decentralized Data. Proc. 20th Int. Conf. Artif. Intell. Stat. AISTATS 2017 (2016).

40. Shokri, R. \& Shmatikov, V. Privacy-Preserving Deep Learning|Proceedings of the 22nd ACM SIGSAC Conference on Computer and Communications Security. ACM Digit. Libr. 1310-1321 (2015).

41. Pathak, M., Rane, S., Sun, W. \& Raj, B. Privacy preserving probabilistic inference with Hidden Markov Models. in ICASSP, IEEE International Conference on Acoustics, 
Speech and Signal Processing - Proceedings 5868-5871 (2011). doi:10.1109/ICASSP.2011.5947696.

42. Chaussabel, D., Pascual, V. \& Banchereau, J. Assessing the human immune system through blood transcriptomics. BMC Biology. 8, (2010).

43. Chaussabel, D. Assessment of immune status using blood transcriptomics and potential implications for global health. Seminars in Immunology. 27, 58-66 (2015).

44. Wei, Z. et al. Large sample size, wide variant spectrum, and advanced machinelearning technique boost risk prediction for inflammatory bowel disease. Am. J. Hum. Genet. 92, 1008-1012 (2013).

45. Stephens, Z. D. et al. Big data: Astronomical or genomical? PLoS Biol. 13, (2015).

46. Dove, E. S. et al. Genomic cloud computing: Legal and ethical points to consider. Eur. J. Hum. Genet. 23, 1271-1278 (2015).

47. Warnat-Herresthal, S. et al. Scalable Prediction of Acute Myeloid Leukemia Using HighDimensional Machine Learning and Blood Transcriptomics. iScience 23, (2020).

48. Zak, D. E. et al. A blood RNA signature for tuberculosis disease risk: a prospective cohort study. Lancet 387, 2312-2322 (2016).

49. Leong, S. et al. Existing blood transcriptional classifiers accurately discriminate active tuberculosis from latent infection in individuals from south India. Tuberculosis 109, 4151 (2018).

50. de Araujo, L. S. et al. Transcriptomic biomarkers for tuberculosis: Evaluation of DOCK9, EPHA4, and NPC2 mRNA expression in peripheral blood. Front. Microbiol. 7, (2016).

51. Verma, S. et al. 'Tuberculosis in advanced HIV infection is associated with increased expression of IFNy and its downstream targets'. BMC Infect. Dis. 18, (2018).

52. Thompson, E. G. et al. Host blood RNA signatures predict the outcome of tuberculosis treatment. Tuberculosis 107, 48-58 (2017).

53. Corman, V. M. et al. Detection of 2019 novel coronavirus (2019-nCoV) by real-time RTPCR. Eurosurveillance 25, (2020).

54. Schulte-Schrepping, J. et al. Suppressive myeloid cells are a hallmark of severe COVID-19. medRxiv 2020.06.03.20119818 (2020) doi:10.1101/2020.06.03.20119818.

55. McKinney, S. M. et al. International evaluation of an Al system for breast cancer screening. Nature 577, 89-94 (2020).

56. Kermany, D. S. et al. Identifying Medical Diagnoses and Treatable Diseases by ImageBased Deep Learning. Cell 172, 1122-1131.e9 (2018).

57. Ardila, D. et al. End-to-end lung cancer screening with three-dimensional deep learning on low-dose chest computed tomography. Nat. Med. 25, 954-961 (2019).

58. Esteva, A. et al. Dermatologist-level classification of skin cancer with deep neural networks. Nature 542, 115-118 (2017).

59. Kaissis, G. et al. A machine learning model for the prediction of survival and tumor subtype in pancreatic ductal adenocarcinoma from preoperative diffusion-weighted imaging. Eur. Radiol. Exp. 3, (2019).

60. Kaissis, G. et al. A machine learning algorithm predicts molecular subtypes in pancreatic ductal adenocarcinoma with differential response to gemcitabine-based versus FOLFIRINOX chemotherapy. PLoS One 14, (2019).

61. Cui, E. et al. Predicting the ISUP grade of clear cell renal cell carcinoma with multiparametric MR and multiphase CT radiomics. Eur. Radiol. 30, 2912-2921 (2020). 
62. $\mathrm{Lu}, \mathrm{H}$. et al. A mathematical-descriptor of tumor-mesoscopic-structure from computedtomography images annotates prognostic- and molecular-phenotypes of epithelial ovarian cancer. Nat. Commun. 10, (2019).

63. Elshafeey, N. et al. Multicenter study demonstrates radiomic features derived from magnetic resonance perfusion images identify pseudoprogression in glioblastoma. Nat. Commun. 10, (2019).

64. Ryffel, T., Dufour-Sans, E., Gay, R., Bach, F. \& Pointcheval, D. Partially Encrypted Machine Learning using Functional Encryption. (2019).

65. Salem, M., Taheri, S. \& Yuan, J.-S. Utilizing Transfer Learning and Homomorphic Encryption in a Privacy Preserving and Secure Biometric Recognition System. Computers 8, 3 (2018).

66. Dahl, M. et al. Private Machine Learning in TensorFlow using Secure Computation. (2018).

67. Shi, W. et al. Deep Learning-Based Quantitative Computed Tomography Model in Predicting the Severity of COVID-19: A Retrospective Study in 196 Patients. SSRN Electron. J. (2020) doi:10.2139/ssrn.3546089.

68. Edgar, R., Domrachev, M. \& Lash, A. E. Gene Expression Omnibus: NCBI gene expression and hybridization array data repository. Nucleic Acids Res. (2002) doi:10.1093/nar/30.1.207.

69. Irizarry R. A. Exploration, Normalization, and Summaries of High Density Oligonucleotide Array Probe Level Data. Biostatistics (2003).

70. Gautier L. Affy--Analysis of Affymetrix GeneChip Data at the Probe Level. Bioinformatiks (2004).

71. Love, M. I., Huber, W. \& Anders, S. Moderated estimation of fold change and dispersion for RNA-seq data with DESeq2. Genome Biol. 15, (2014).

72. Dobin, A. et al. STAR: Ultrafast universal RNA-seq aligner. Bioinformatics 29, 15-21 (2013).

73. Zwiener, I., Frisch, B. \& Binder, H. Transforming RNA-Seq data to improve the performance of prognostic gene signatures. PLoS One 9, (2014).

74. Efron, B. Bootstrap Methods: Another Look at the Jackknife. Ann. Stat. 7, 1-26 (1979).

75. Pedregosa, F. et al. Scikit-learn: Machine Learning in Python. Journal of Machine Learning Research. 12, (2011). 